\title{
ARTICLE
}

\section{Entamoeba histolytica stimulates the alarmin molecule HMGB1 from macrophages to amplify innate host defenses}

\author{
Sharmin Begum ${ }^{1}$, France Moreau ${ }^{1}$, Aralia Leon Coria ${ }^{1}$ and Kris Chadee ${ }^{1}$
}

\begin{abstract}
Even though Entamoeba histolytica (Eh)-induced host pro-inflammatory responses play a critical role in disease, we know very little about the host factors that regulate this response. Direct contact between host cell and Eh signify the highest level of danger, and to eliminate this threat, the host immune system elicits an augmented immune response. To understand the mechanisms of this response, we investigated the induction and release of the endogenous alarmin molecule high-mobility group box 1 (HMGB1) that act as a pro-inflammatory cytokine and chemoattractant during Eh infection. Eh in contact with macrophage induced a dose- and time-dependent secretion of HMGB1 in the absence of cell death. Secretion of HMGB1 was facilitated by Eh surface Gal-lectinactivated phosphoinositide 3-kinase and nuclear factor-KB signaling and up-regulation of histone acetyltransferase activity to trigger acetylated HMGB1 translocation from the nucleus. Unlike lipopolysaccharide, Eh-induced HMGB1 release was independent of caspase-1-mediated inflammasome and gasdermin D pores. In vivo, Eh inoculation in specific pathogen-free but not germ-free mice was associated with high levels of pro-inflammatory cytokines such as tumor necrosis factor- $\alpha$, interleukin-1 $\beta$, and keratinocyte-derived chemokine, which was suppressed with HMGB1 neutralization. This study reveals that Eh-induced active secretion of the HMGB1 plays a key role in shaping the pro-inflammatory landscape critical in innate host defense against amebiasis.
\end{abstract}

Mucosal Immunology (2020) 13:344-356; https://doi.org/10.1038/s41385-019-0233-6

\section{INTRODUCTION}

Entamoeba histolytica (Eh) is an extracellular invasive protozoan parasite and the causative agent of amebiasis. Eh infection is acquired by the ingestion of infective cysts contaminated with food or water. The majority of the Eh infection is tolerated in the lumen without disease symptoms (asymptomatic infection). For reasons still unknown, $\sim 10 \%$ of Eh infection occasionally breaches innate mucosal barriers in the gut and invades the lamina propria and disseminate through the portal vein to the liver where it causes liver abscesses. $^{1-3}$ The exact factors responsible for this infrequent parasite invasion are not fully understood. It is generally believed that asymptomatic/symptomatic infection depends on parasite virulent factors and their interaction as well as the quality of the host innate immune responses. ${ }^{4}$ When $E h$ invades, the host innate immune system triggers a florid pro-inflammatory response characterized by interleukin-1 $\beta$ (IL-1 $\beta$ ) and tumor necrosis factor- $\alpha$ (TNF-a) release, which are thought to exacerbate disease. ${ }^{2}$ In disease pathogenesis, one of the unsolved question is, how does the immune response escalate at the site of Eh invasion? At the parasite level, Eh Gal/GalNAc lectin (Gal-lectin)-mediated adherence to host cells triggers innate immune responses that contribute in disease pathogenesis. ${ }^{4,5}$ Direct adherence between host cells and Eh specify the presence of an instant threat and the immune system needs to be quickly boosted at the site of active infection to abolish and prevent further spread of the parasite. ${ }^{5}$ At present, there is a knowledge gap in our understanding of the mechanism behind the augmented inflammatory responses triggered during $E h$ invasion.
Host immune responses are another critical determinant of Eh infection to induce disease. Macrophages are considered the key innate immune cell present in the lamina propria that respond against invasive $E h$ to actively secrete pro-inflammatory mediators to recruit additional immune cells to combat infection. ${ }^{6,7}$ Macrophage produce high levels of TNF- $a$ that is associated with increased diarrheal disease and interferon- $\gamma$ (IFN- $\gamma$ ) and nitric oxide to protect from infection. ${ }^{7-9}$ Eh interaction up-regulates Tolllike receptor (TLR2 and TLR4) expression in macrophages and activate nuclear factor-KB (NF-KB) signaling pathway to induce pro-inflammatory cytokines. ${ }^{10,11}$ Recently, we have unraveled that direct contact between $E h$ and macrophage instigates multiple intercellular mechanisms. ${ }^{4,5}$ In particular, engagement between $E h$ cysteine protease 5 (EhCP-A5) RGD sequence to $a_{5} \beta_{1}$ integrin on macrophage activates the NLRP3 inflammasome, which leads to $\mathrm{IL}-1 \beta$ secretion. ${ }^{5}$ In addition, direct interaction at the intercellular junction by EhCP-A1- and EhCP-A4-activated caspase-6-mediated degradation of cytoskeletal proteins and potentiated IL-1 $\beta$ release. $^{4}$ More recently, we found that Eh-activated caspase- 4 interact with caspase- 1 in a protein complex to enhance cleavage of caspase-1 CARD domain to potentiate IL-1 $\beta$ secretion by cleaving gasdermin $\mathrm{D}$ (GSDMD) N-terminal pore-forming fragment. ${ }^{12}$ These studies reinforce the requirement of direct contact between $E h$ and host immune cell to initiate various cellular mechanisms to induce pro-inflammatory responses. In Eh infection, very few parasites invade the lamina propria and come in direct contact with macrophages, which raises the question, how does innocent bystander immune cells "sense" invading Eh?

${ }^{1}$ Department of Microbiology, Immunology and Infectious Diseases, Snyder Institute for Chronic Diseases, University of Calgary, Calgary, AB, Canada Correspondence: Kris Chadee (kchadee@ucalgary.ca)

Received: 13 June 2019 Revised: 22 October 2019 Accepted: 7 November 2019

Published online: 26 November 2019 
Histological studies characterize inflammation following microerosions of the colonic epithelium prior to $E h$ invasion into the lamina propria. ${ }^{13} \mathrm{Eh}$ invasion always induces a prompt robust proinflammatory response, and this signature event led us to hypothesize that early alarmin signals triggers the assembly of adjacent immune cells to the site of infection to augment the secretion of pro-inflammatory cytokines and chemokines. The aim of this study was to determine the earliest alarmin signal released by host cells following contact with Eh. We found that the endogenous alarmin molecule high-mobility group box 1 (HMGB1) was activated and released from macrophages during early encounter with live Eh. Eh Gal-lectin contact with macrophages activated phosphoinositide 3-kinase (PI3K) and NF-KB signaling pathways that up-regulated the acetylation and release of HMGB1. Unlike lipopolysaccharide (LPS), the extracellular translocation of HMGB1 was independent of inflammasome activation. In vivo, Eh-induced pro-inflammatory responses were suppressed by HMGB1 neutralization. This study reveals that Ehinduced alarmin molecules can shape the magnitude of the proinflammatory response that is critical in innate host defense against amebiasis.

\section{RESULTS}

Entamoeba histolytica induces rapid and early secretion of the alarmin molecule HMGB1

We have previously shown that direct contact between Eh macrophages triggers inflammasome activation and caspase-1/4/ 6-mediated IL-1 $\beta$ release. ${ }^{4,5,12,14}$ To determine how bystander non Eh-contacted immune cells are activated and/or recruited to amplify pro-inflammatory responses at the site of infection, we quantified the secretion of the well-known endogenous alarmin protein HMGB1. To do this, human THP-1 and mouse bone marrow-derived macrophages (BMDMs) were exposed to graded dosages of $E h$ and found that 20:1 macrophage to Eh ratio was the sub-optimal dose that triggered robust HMGB1 protein release in the supernatant (SN) by western blot analysis (Fig. 1a). With this sub-optimal dose, Eh elicited the release of HMGB1 as early as $5 \mathrm{~min}$ in a time-dependent manner that peaked at $30 \mathrm{~min}$ in both THP-1 and BMDM (Fig. 1b, c).

HMGB1 is a pre-formed protein abundantly expressed in the nucleus, and basally (-ve control) HMGB1 remains within cell lysates (LYS). To determine if Eh could stimulate HMGB1 secretion in cells other than macrophages, human colonic T84 epithelial cells were stimulated with Eh for 5 to $30 \mathrm{~min}$ and with different multiplicity of infection that showed time- and dose-dependent HMGB1 secretion similar to macrophages (Supplementary Fig. S1a/b). In comparison, the kinetics of HMGB1 secretion in response to the positive control LPS $(100 \mathrm{ng} / \mathrm{ml})$ occurred only after $4 \mathrm{~h}$ (Supplementary Fig. S1c). As HMGB1 can be released passively from necrotic cells, we determined the levels of cytotoxicity in THP-1 and BMDM cells exposed to Eh by measuring lactate dehydrogenase (LDH) and found $<5 \%$ cell death up to 20 min of Eh stimulation (Fig. 1b, c). As additional controls, lysed Eh, Eh SN, and THP-1 SNs (-ve control) showed no detectable HMGB1 as compared to recombinant HMGB1 (rHMGB1; Supplementary Fig. S1d). Eh stimulation of THP-1 cells also did not upregulate HMGB1 messenger RNA (mRNA) transcripts (Supplementary Fig. S1e) comparable to a previous study. ${ }^{15}$ These results confirmed that $E h$ induces rapid and time-dependent secretion of HMGB1 without cell death in immune and epithelial cells.

Cytoplasmic translocation of HMGB1 in macrophages exposed to and in contact with $E h$

HMGB1 is a non-histone nuclear-binding protein that predominantly resides within the nucleus of the cell. ${ }^{16}$ To assess the translocation of nuclear HMGB1 into the cytoplasm and subsequently in the extracellular space, we performed immunostaining of HMGB1. BMDMs were stimulated with $E$ for $10 \mathrm{~min}$ and the subcellular localization of HMGB1 was determined as compared to non-stimulated cells (Fig. 2a). In comparison with nonstimulated cells, Eh-stimulated macrophages showed significant translocation of HMGB1 into the cytoplasm as distinct puncta randomly distributed throughout the cytoplasm [Fig. 2a, b, green specks (white arrows, panels 1 and 2)]. In response to $E h$ in direct contact with macrophages, cytoplasmic HMGB1 was also distributed in the cytoplasm and was not polarized at the point of Eh contact (Fig. 2a, panels 3 and 4). These results demonstrate that HMGB1 translocate from the nucleus to the cytoplasm and are destined to export into the extracellular space in response to $E h$.

HMGB1 release is dependent on contact with live Eh via cell surface-bound Gal-lectin

To determine the parasite component that triggered the release of HMGB1, macrophages were stimulated with live Eh or with different components of $E h$, including concentrated secreted proteins (SPs, $50 \mu \mathrm{g}$ ), equal amount of whole Eh lysates, and cytoplasmic and membrane components for 30 and $60 \mathrm{~min}$. Only live $E h$ and not $E h$ components stimulated the release of HMGB1 (Fig. 3a). Even macrophages stimulated up to $16 \mathrm{~h}$ with sub-cellular fractions of $E h$ did not induce the secretion of HMGB1, whereas LPS (100 ng/ml), a known inducer of HMGB1, did (Fig. 3b). Similar to Eh-induced inflammasome and inflammatory caspase activation, ${ }^{12,14}$ HMGB1 secretion from macrophages required live $E h$ in direct contact with cells. These results prompted us to determine if Eh Gal-lectin-mediated contact with macrophages was required to trigger the release of HMGB1 from the nucleus. To interrogate this, macrophages were exposed to $E h$ in the presence of $55 \mathrm{mM}$ exogenous galactose to competitively block Eh Gal-lectin interaction on the macrophage surface. As predicted, galactose significantly abrogated HMGB1 release as compared to Eh-only stimulated cells (Fig. 3c). Eh Gal-lectin is highly immunogenic and enhances the transcription of pro-inflammatory cytokines. ${ }^{10,14,17}$ Thus, to determine if native Eh Gal-lectin $(500 \mathrm{ng} / \mathrm{ml})$ could stimulate HMGB1, macrophages were stimulated up to $4 \mathrm{~h}$ and compared to LPS $(100 \mathrm{ng} / \mathrm{ml})$. In comparison to LPS, native Eh Gal-lectin modestly stimulated HMGB1 release in a time-dependent fashion (Fig. 3d). These results verify that native Gal-lectinmediated interaction with macrophages from live $E h$ was critical for rapid and early extracellular release of HMGB1. As Gal-lectin and membrane-bound EhCP-A5 play critical roles in activating caspase-1/4 and the NLRP3 inflammasome, we tested if both virulent factors played a role in HMGB1 release. Interestingly, macrophages exposed to $E h C P-A 5^{-}$showed no difference in HMGB1 secretion as compared to WT Eh (Supplementary Fig. S2a). During Eh-macrophage contact, remarkable cytoskeletal rearrangement along with actin remodeling occurs, ${ }^{5}$ and as expected, cells pre-treated with cytochalasin D significantly reduced $\mathrm{HMGB} 1$ release, indicating the requirement for live $E$ hmediating signal transduction (Supplementary Fig. S2b).

Eh induces the acetylation and active secretion of HMGB1 HMGB1 can release actively from stimulated immune cells or passively from necrotic or damaged cells. Active secretion is mediated by the acetylation of lysine residues present in the two nuclear localization sequence (NLS). ${ }^{18}$ As Eh-exposed macrophages showed negligible cell death $(<2 \%$; Fig. 1 b, c) at early time points (up to $10 \mathrm{~min}$ ), we next explored how HMGB1 is secreted by macrophages. By western blot, we observed the presence of doublet bands after $20 \mathrm{~min}$ exposure with $E h$ as compared to the earlier time points $(2-10 \mathrm{~min})$ due to molecular weight shifting. This suggests that the early release of HMGB1 occurred through the active secretion pathway of lysine residue acetylation specified by the higher molecular weight band 

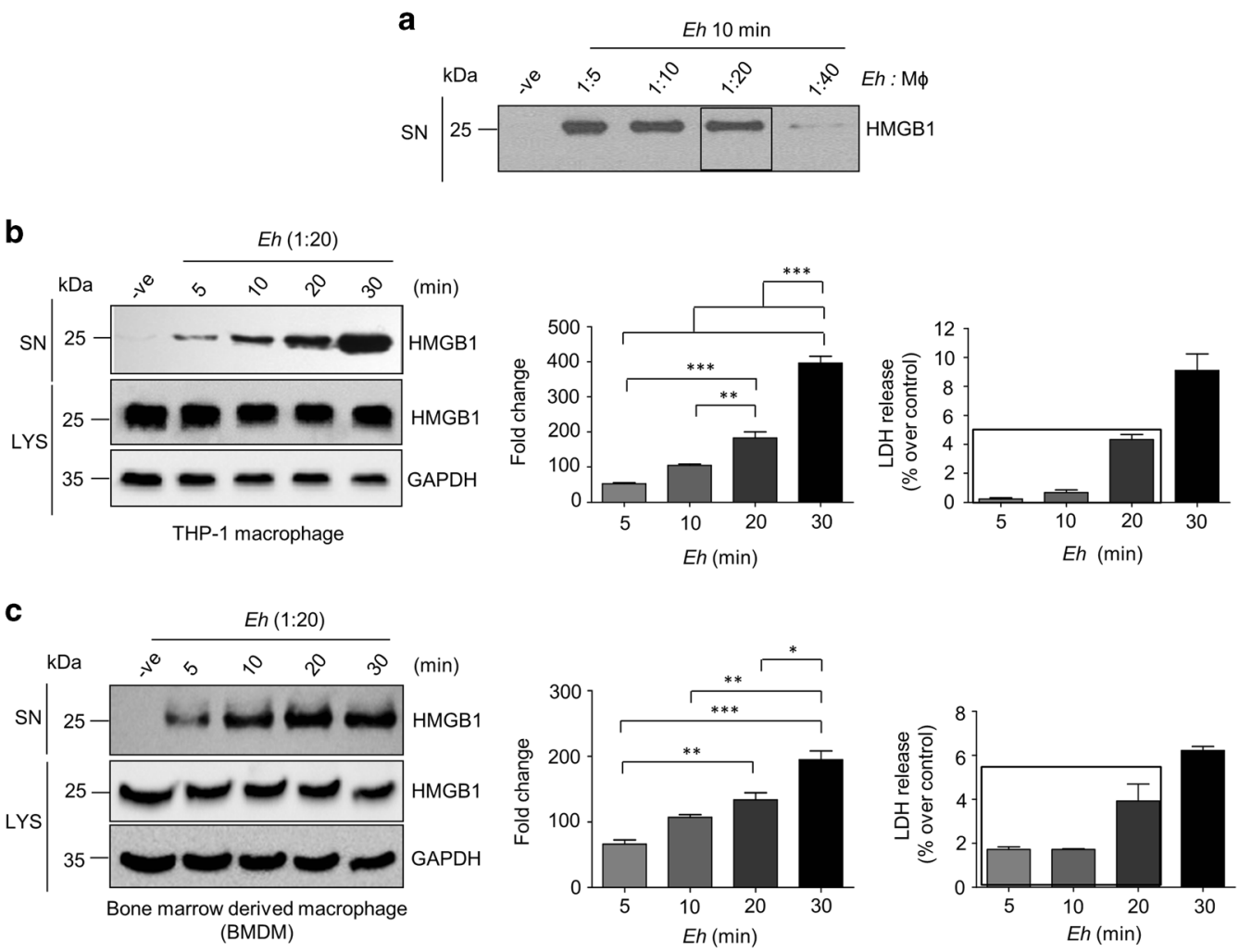

Fig. 1 Entamoeba histolytica induces rapid and early secretion of the alarmin molecule HMGB1 in macrophages. a THP-1 macrophages were stimulated with different $E h$ to macrophage ratios to identify the sub-optimal infection dose. $\mathbf{b}$ THP- 1 macrophages and $\mathbf{c}$ bone marrowderived macrophages (BMDMs) were stimulated with Eh at a (20:1 ratio) for 5 to 30 min. Equal amounts of cell culture supernatants (SNs) and cell lysates (LYS) were loaded on to the SDS-PAGE gel for HMGB1 detection. Densitometric analysis was done to quantify time-dependent HMGB1 secretion in THP-1 and BMDM cells, respectively. Cell death in b THP-1 and c BMDM cells were measured by the release of lactate dehydrogenase (LDH assay) following Eh stimulation normalized to non-stimulated negative controls (basal cell death). Data are representative of three independent experiments and statistical significance was determined by one-way ANOVA, followed by post hoc Bonferroni test. ${ }^{*} P<0.05,{ }^{*} P<0.01$, and ${ }^{* * *} P<0.001$. Bars represent mean $\pm \mathrm{SEM}$.

(acetylated form) observed up to $10 \mathrm{~min}$. Subsequently, the presence of the second band of lower molecular weight (nonacetylated form) appeared after 20 to $40 \mathrm{~min}$ (Fig. 4a). To confirm this, immunoprecipitation (IP) assays targeting the acetylated lysine residues showed the presence of acetylated HMGB1 within 10 min of Eh stimulation as compared to nonstimulated and isotype controls (Fig. 4b, top panel). Western blot with the SN from this experiment confirmed Eh-mediated secretion of HMGB1 at that time point (Fig. 4b, bottom panel). As the acetylation of nuclear proteins are typically mediated by histone acetyltransferases (HAT), ${ }^{19}$ we assessed the requirement for HAT activity using the broad-spectrum HAT inhibitor, anacardic acid (AA). As predicted, inhibition of HAT activity with AA significantly diminished $E h$-induced secretion of HMGB1 as compared to Eh-only stimulation (Fig. 4c). To corroborate these findings, we performed immunostaining of HMGB1 protein in macrophages in direct contact with $E h$ in the presence or absence of AA and observed significant inhibition of cytoplasmic translocation of nuclear HMGB1 as compared to Eh-only stimulation (Fig. 4d, e). We also investigated HAT activity following Eh stimulation at different time points using a HAT activity assay and observed a trend of increased enzymatic activity compared to the negative control (Fig. 4f). Inhibition with AA reduced HAT activity (Fig. 4f) and corroborated the results of the western blot and immunostaining for HMGB1 release (Fig. 4c, d). These results confirm that $E h$ induces the acetylation of HMGB1 that subsequently mediated active secretion into the cytoplasm of macrophages.
$\mathrm{PI} 3 \mathrm{~K}$ and NF-KB signaling are required for acetylation and active secretion of HMGB1 in response to $E h$

We next investigated the mechanism whereby the Gal-lectin signaling pathway induced the acetylation and release of HMGB1 (Fig. 3c, d). We have previously shown that Gal-lectin activates the NF-KB signaling pathway in macrophages, ${ }^{10,17}$ and Eh-induced mucin secretion is initiated by the PI3K signaling cascade. ${ }^{20}$ In response to $E h$, activation of the PI3K signaling pathway in macrophages was evident by the appearance of phosphorylated (p)-PI3K as early as $2 \mathrm{~min}$ of stimulation with maximum p-PI3K at 10 min (Fig. 5a). Similarly, the NF-KB signaling pathway was activated by the presence of p-IKB (Fig. 5a) and p-P65 in cytoplasmic extracts (Fig. 5b). Exogenous galactose-mediated competitive inhibition of Gal-lectin binding with macrophages markedly diminished p-PI3K, p-IKB, and HMGB1 secretion as compared to only Eh (Fig. 5c). Based on these findings, we then investigated if native Gal-lectin could activate the PI3K and NF-KB signaling pathway and observed a time-dependent increase in $\mathrm{p}$ PI3K and $\mathrm{p}$-IKB level (Supplementary Fig. S3). To explore if these pathways were important in inducing active secretion of HMGB1, THP-1 macrophages were stimulated with $E h$ in the presence or absence of PI3K inhibitors Wortmannin and Ly294002, which significantly reduced Eh-induced HMGB1 secretion with subsequent inhibition of p-PI3K (Fig. 5d). We next investigated the role of the NF-KB signaling pathway in Eh-induced HMGB1 release in the presence or absence of the NF-KB pharmacological inhibitor Bay-11-7082, and it significantly abrogated HMGB1 release and $p-$ IKB (Fig. 5e). These results indicate that both PI3K and NF-KB 
a
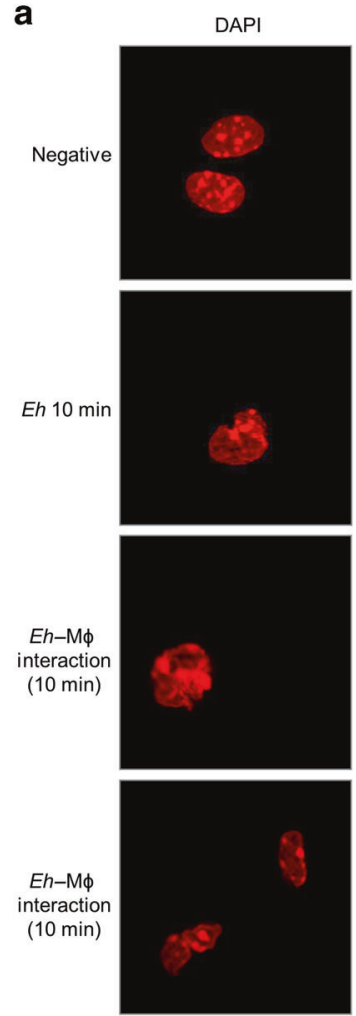

HMGB1
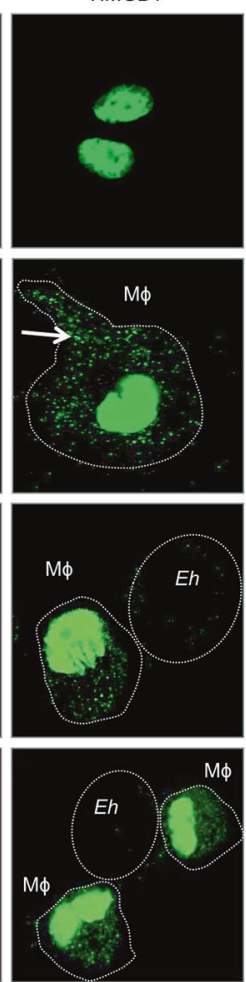

DAPI-HMGB1
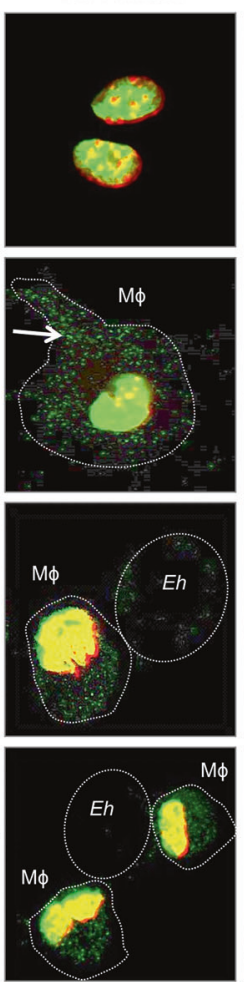

DIC
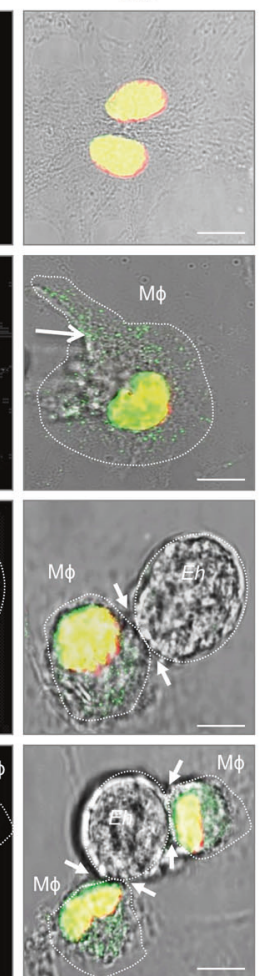

b

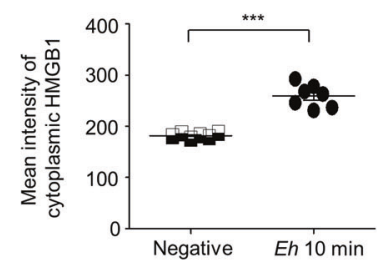

Fig. 2 Eh in contact with macrophage induces cytoplasmic translocation of HMGB1. BMDMs were stimulated with Eh for 10 min and translocation of nuclear HMGB1 was measured by immunofluorescence analysis. a Representative images of HMGB1 immunostaining in control and Eh-exposed macrophages from three independent experiments (scale bar: $10 \mu \mathrm{m}$ ). The white arrows in the DIC images show the point of contact of $E h$ with macrophages $(M \phi)$. b The mean intensity of the cytoplasmic HMGB1 staining in control and Eh-infected macrophages are shown as mean \pm SEM. Statistical significance was determined by $t$ test. ${ }^{* * *} P<0.001$.

signaling pathways were activated by Eh Gal-lectin in contact with macrophages that regulated HMGB1 release.

As active secretion of HMGB1 require acetylation within NLS to lose its interaction with the nucleus, allowing it to translocate into the cytoplasm, ${ }^{18}$ we next interrogated whether PI3K and NF-KB signaling was required for the acetylation of HMGB1. To do this, macrophages were stimulated with Eh for $10 \mathrm{~min}$ in the presence or absence of the HAT inhibitor AA (as control), Ly294002, and Bay-11-7082. This was followed by IP using acetyl lysine antibody to pull down the acetylated protein, and by immunoblot, acetylated HMGB1 was detected. As expected, Eh-induced acetylation of HMGB1 was markedly reduced in the presence of AA (Fig. 5f). Similarly, inhibitors of PI3K, Ly294002 (Fig. 5g), and NF$\mathrm{KB}$, Bay-11-7082 (Fig. 5h), significantly reduced the acetylation of HMGB1. Taken together, these results demonstrate that along with HAT activity, both $\mathrm{PI} 3 \mathrm{~K}$ and NF-KB signaling pathways are necessary for the acetylation and secretion of $\mathrm{HMGB} 1$ in response to Eh.

Inflammasome and GSDMD-independent extracellular release of HMGB1 in response to $E h$

HMGB1 lacks a leader peptide sequence required for the classical Golgi apparatus/endoplasmic reticulum-dependent secretion; thus, the mechanism of active HMGB1 secretion from cells remains unknown. Previous studies have shown that during endotoxemia, inflammasome activation mediates the release of HMGB1 from activated immune cells. ${ }^{21,22}$ We have shown that $E h$ induces caspase-1-mediated NLRP3 inflammasome activation in macrophages. ${ }^{5,14}$ To determine if inflammasome components are involved in Eh-induced HMGB1 release, BMDMs isolated from wild-type (WT) and Caspase $1^{-1-}$ mice were stimulated with Eh for 10-30 min. Eh stimulated the secretion of HMGB1 in both WT and
Caspase $1^{-1-}$ macrophages, whereas in response to the potent inflammasome activator LPS + nigericin, secretion was completely dependent on caspase-1 (Fig. 6a, b). We next quantified the requirement for the adaptor protein apoptosis-associated specklike protein containing a CARD (ASC), which bridges the interaction between the NLR proteins and caspase-1. WT THP-1 and ASC-deficient THP-1 macrophages were stimulated with Eh for different time points and HMGB1 release was not affected by ASC deficiency; however, with LPS + nigericin stimulation, HMGB1 levels were absent supporting the previous observation of ASC dependency with endotoxemia (Fig. 6c, d). As Eh can activate the NLRP3 inflammasome, ${ }^{5}$ macrophages from WT and NLRP3 CRISPR/ Cas9-knockout (KO) THP-1 macrophages were stimulated with Eh for different time points and it also had no effect on HMGB1 secretion, whereas with LPS + nigericin, NLRP3 was required for $\mathrm{HMGB} 1$ secretion (Fig. 6e, f). Similarly, there was no difference observed in $E$-induced HMGB1 secretion from WT and $\mathrm{NIrp3}^{-1-}$ BMDM cells (Supplementary Fig. S4). The contrast in the requirements between LPS + nigericin and Eh-induced HMGB1 secretion indicates that early HMGB1 secretion with Eh is independent of caspase-1-mediated inflammasome activation and pyroptotic cell death.

From the above studies, even though $E h$-induced HMGB1 secretion was independent of caspase-1, ASC, and NLRP3 inflammasome activation (Fig. 6a-e), it does not exclude the possibility that HMGB1 may be exported from cells via membrane pores. We have recently shown that $E$-induced caspase-1/4 activation cleaved GSDMD, which oligomerized in the plasma membrane to create lipid bilayer pores that caused the secretion of IL-1 $1 .^{12} \mathrm{IL}-1 \beta$ is expressed as a precursor protein, and similar to HMGB1, it lacks signal peptide sequence for classical Golgi/endoplasmic reticulum secretion. ${ }^{23}$ GSDMD pores serve as channel for the extracellular 


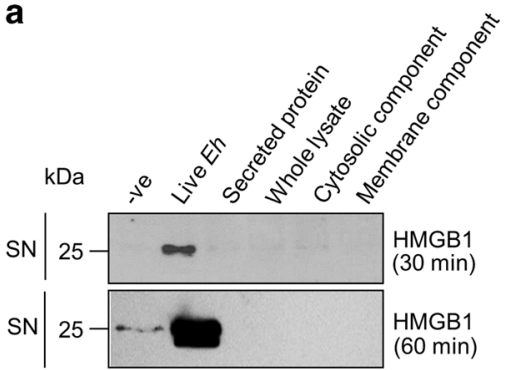

C

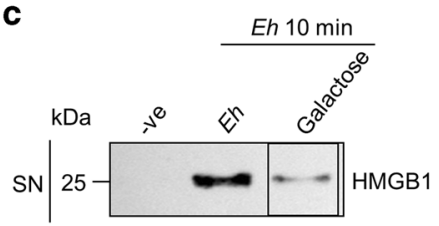

d

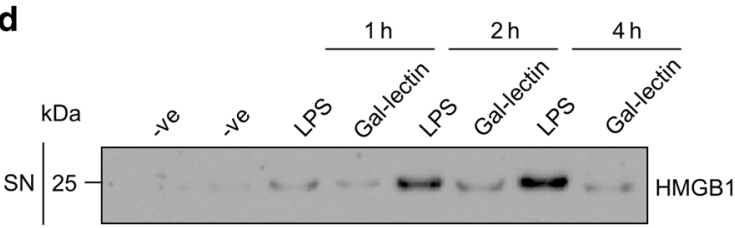

b
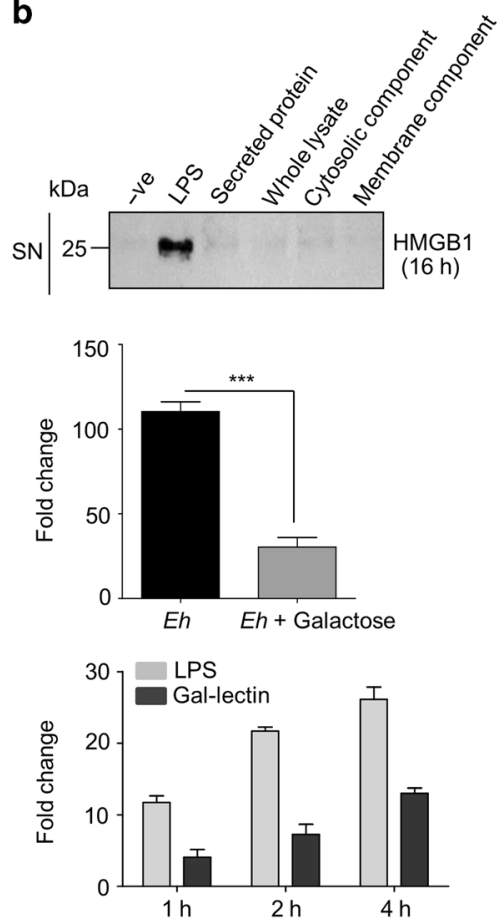

Fig. 3 HMGB1 release requires live Eh Gal-lectin-mediated contact with macrophages. a THP-1 cells were stimulated for 30 and 60 min with live $E h(20: 1$ ratio), secreted proteins $(S P s, 50 \mu \mathrm{g})$ from $E h$, whole lysates of $E h$, and cytoplasmic and membrane components of an equal amount of Eh. Equal amounts of cell culture supernatant (SN) samples were loaded onto the SDS-PAGE gel for HMGB1 detection. b THP-1 cells were stimulated up to $16 \mathrm{~h}$ with LPS $(100 \mathrm{ng} / \mathrm{ml})$ and Eh sub-cellular fractions and secretion of HMGB1 protein was assessed. c Eh was pretreated for $5 \mathrm{~min}$ with $55 \mathrm{mM}$ D-galactose before incubation with macrophages. THP-1 cells were stimulated with either galactose-treated Eh or Eh only for $10 \mathrm{~min}$ to assess HMGB1 secretion. Densitometric analysis showed significant inhibition of HMGB1 secretion following galactose treatment. d THP-cells were treated with native Eh Gal-lectin $(500 \mathrm{ng} / \mathrm{ml})$ or LPS $(100 \mathrm{ng} / \mathrm{ml})$ for different time points $(1-4 \mathrm{~h})$ and HMGB1 secretion was determined by western blot. Data are from three independent experiments and statistical significance was determined by $t$ test ${ }^{* *} P<0.001$. Bar represent mean \pm SEM.

transport of IL-1 from hyperactive live macrophages in the absence of pyroptosis. ${ }^{24,25}$ To interrogate how HMGB1 protein is exported outside the cell, we investigated whether GSDMD pores were a likely candidate. To explore this, both WT THP-1 and GSDMD CRISPR/Cas9-KO cells were stimulated with Eh for different time points and observed no differences in the HMGB1 secretion (Fig. 6g). In marked contrast, under the same conditions, cells were stimulated with LPS + nigericin, and HMGB1 secretion was completely dependent on the presence of GSDMD pores (Fig. 6h). As GSDMD CRISPR/Cas9 cells stimulated with Eh have slightly higher cell death, ${ }^{12}$ we determined the acetylation status of secreted HMGB1 and found equal levels of acetylated HMGB1 in both WT and GSDMD CRISPR/Cas9-KO cells (Fig. 6i, upper panel). Moreover, total HMGB1 protein present in cell lysates showed no differences within the groups (Fig. 6i, bottom panel). GSDMD pores are key mediators of pyroptotic cell death, and with LPS + nigericin, rapid activation of inflammasome leads to pyroptosis, which release HMGB1, whereas in response to $E h$, secretion of cytoplasmic-acetylated HMGB1 appears to be facilitated by an unknown mechanism independent of inflammasome and GSDMD pores.

In vitro silencing and in vivo neutralization of HMGB1 reduces proinflammatory cytokine expression

As global HMGB1-KO mice die within $24 \mathrm{~h}$ of birth, we silenced HMGB1 in THP-1 macrophages by small interfering RNA (siRNA) along with scrambled siRNA as controls. HMGB1 silencing reduced $\sim 50 \%$ basal protein (Fig. 7a) and mRNA transcript expression (Fig. $7 \mathrm{~b}$ ), and in response of $E$, markedly reduced the secretion of HMGB1 (Fig. 7c). Secreted HMGB1 couples to cell surface receptors (RAGE and TLR4) to upregulate pro-inflammatory cytokines in an autocrine and/or paracrine fashion. ${ }^{26}$ Thus, to explore the effect of HMGB1 siRNA silencing on pro-inflammatory cytokine expression, macrophages were stimulated with $E h$ and TNF-a mRNA expression was determined. As predicted, TNF-a mRNA expression was significantly decreased in HMGB1-silenced cells in response to $E h$ as compared to scrambled siRNA controls (Fig. 7d). As controls, to confirm the pro-inflammatory inducer function of HMGB1, exogenously added rHMGB1 alone or in the presence of $E h$ and/ or native Gal-lectin significantly enhanced TNF-a expression synergistically (Fig. 7e).

To quantify a role for HMGB1 in intestinal amebiasis, we used a short-term mouse colonic loop model of infection. ${ }^{27}$ Eh inoculation in colonic loops significantly increased the secretion of HMGB1 in the luminal contents as compared to controls inoculated with phosphate-buffered saline (PBS) (Fig. 7f). To determine if $E$-induced HMGB1 upregulated pro-inflammatory responses, animals were injected intraperitoneally (i.p.) with neutralizing HMGB1 or with an irrelevant IgG control antibody before $E h$ inoculation into colonic loops. While neutralization of HMGB1 showed a decreasing trend in myeloperoxidase (MPO) activity (not significant; Fig. 7g), pro-inflammatory cytokine and chemokine transcripts (IL-1 $\beta$, TNF- $\alpha$, keratinocyte-derived chemokine $(K C)$, and monocyte chemoattractant protein-1) were significantly reduced (Fig. 7h-k). Neutralization of HMGB1 did not inhibit epithelial barrier permeability or luminal exudate secretions in colonic loops inoculated with Eh (Supplementary Fig. S5a/b). Consistent with our previous studies, ${ }^{27}$ we did not observe a significant increase in inflammatory cellular infiltrates in histological sections. Taken together, both in vitro silencing of HMGB1 and in vivo neutralization of HMGB1 showed decreased pro-inflammatory cytokine/chemokine expression in response to 
a

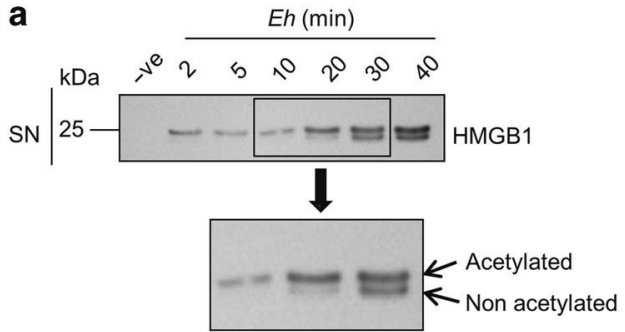

d
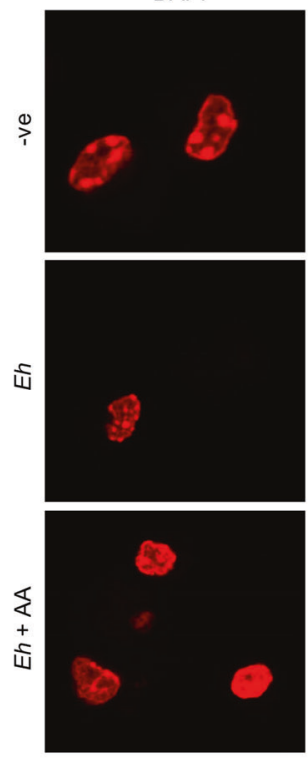

b

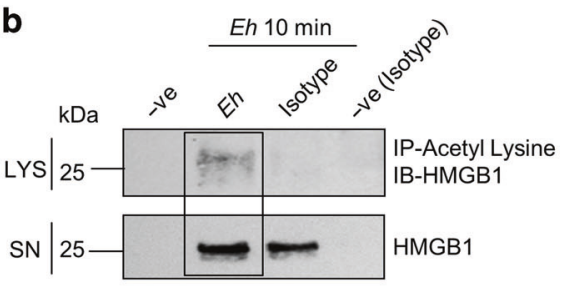

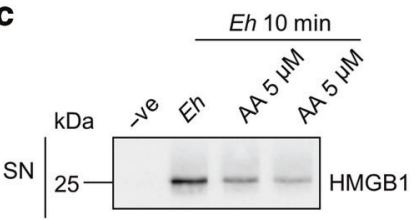
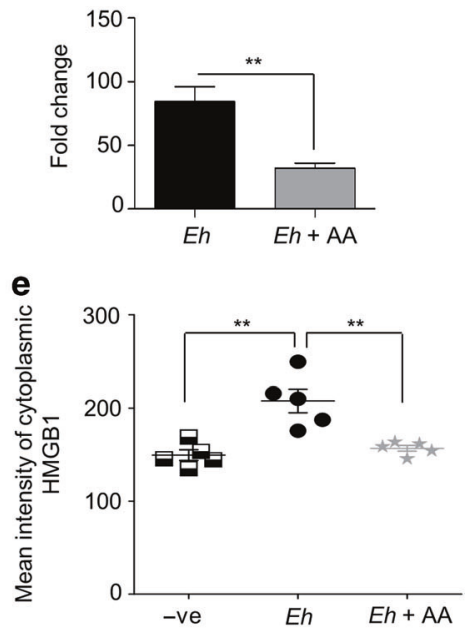

f

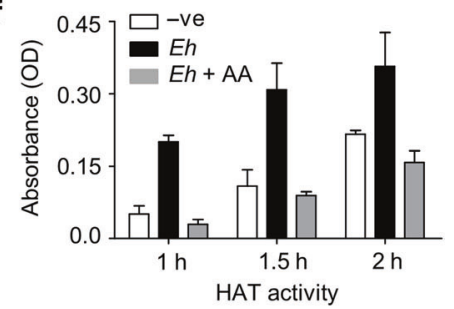

Fig. 4 Active secretion of HMGB1 following Eh contact with macrophages at early time points. a THP-1 cells were incubated with Eh for increasing times at 20:1 ratio and equal amounts of cell culture supernatant (SN) samples were loaded onto the SDS-PAGE gel to detect HMGB1. b Immunoprecipitation assay done with acetyl lysine antibody and immunoblotted with HMGB1 to detect the acetylated form of HMGB1. c THP-1 cells were pre-treated 30 min with $5 \mu \mathrm{M}$ anacardic acid (AA) and then stimulated with Eh for 10 min to detect HMGB1 level. d Immunofluorescence analysis of HMGB1 was done in the presence or absence of AA with Eh after 10 min incubation with macrophages. Representative images from three independent experiments were shown (scale bar: $10 \mu \mathrm{m}$ ). e Quantification of the mean cytoplasmic intensity of HMGB1 shown as mean \pm SEM. $\mathbf{f}$ HAT activity assay was performed following Eh stimulation in the absence or presence of AA. Data are representative of three independent experiments. ${ }^{* *} P<0.001 .{ }^{* *} P<0.01$.

Eh, suggesting that HMGB1 is an early critical mediator that amplifies pro-inflammatory cytokine/chemokine responses in innate host defense against Eh.

Secretion of HMGB1 in response to $E$ is microbiota dependent Gut microbiota modulates bone marrow processes required for the development of immune effector cells, such as neutrophils and inflammatory macrophages, and play a role in establishment of a balanced immune system. ${ }^{28}$ We have recently shown in germfree (GF) mice that, microbiota is required to shape and educate host pro-inflammatory responses against $E h$ infection. ${ }^{29}$ To interrogate the role of microbiota in the regulation of HMGB1 associated with acute inflammation, we quantified secretion of HMGB1 from luminal contents of loops inoculated with Eh in specific pathogen-free (SPF) and GF mice. In response to Eh, luminal contents from SPF showed significantly higher levels of HMGB1 that was completely absent in GF mice and PBSinoculated controls (Fig. 8a). Similarly, BMDM derived from GF mice, but not SPF mice, stimulated with Eh produced less HMGB1 (Fig. 8b). This was not due to reduced total HMGB1 protein content as cell lysates from both SPF and GF BMDM contained equal amounts of protein (Fig. $8 \mathrm{~b}$ ). In accordance with the absence of HMGB1 in the luminal content of GF mice in response to $E h$, mRNA transcripts for IL-1 $\beta$, TNF-a, and KC were also significantly reduced as compared to SPF mice (Fig. 8c-f). These results suggest that microbiota-dependent immune development is required for the secretion of HMGB1 against Eh. A conceptual schematic figure to illustrate how HMGB1 is regulated in response to $E h$ is proposed in Fig. 9.

\section{DISCUSSION}

This current study presents a mechanism by which the extracellular parasite $E$ interacts with host cells via Gal-lectin to induce secretion of the alarmin molecule HMGB1 that escalates inflammatory responses. Eh triggers robust pro-inflammatory responses along with immune cell infiltration to the site of invasion, which play a key role in disease pathogenesis. Our studies address a critical but overlooked feature of Eh pathogenesis: how does bystander non-contacted immune cells sense invading Eh and infiltrate at the site of infection? HMGB1 is a prototypical endogenous danger or alarmin signal for bystander cells when released from activated or necrotic cells. We have shown that coupling of $E h$ Gal-lectin with macrophages activated $\mathrm{PISK}$ and NF-KB signaling pathways that induced acetylation and translocation of HMGB1 from the nucleus to the cytoplasm and subsequently exported outside the cell. Interestingly, in contrast to endotoxemia, Eh-induced HMGB1 release was observed prior to 
a

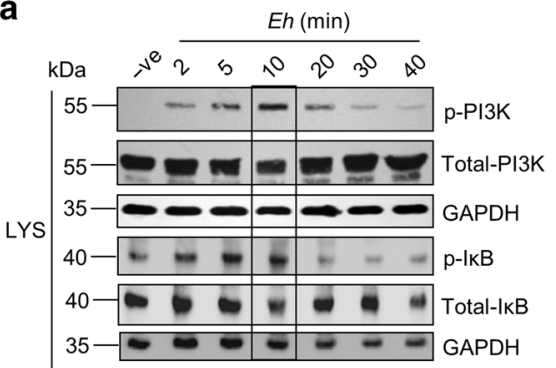

b

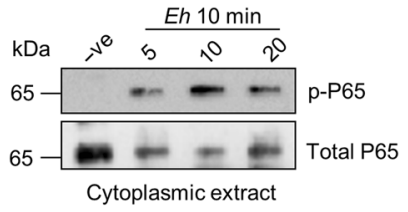

C

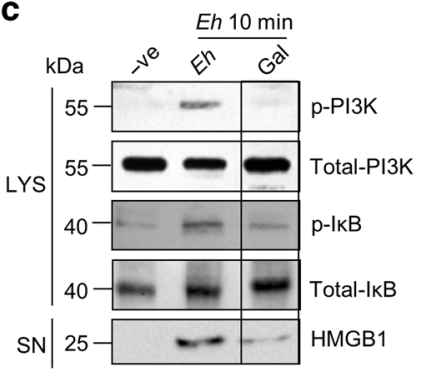

d
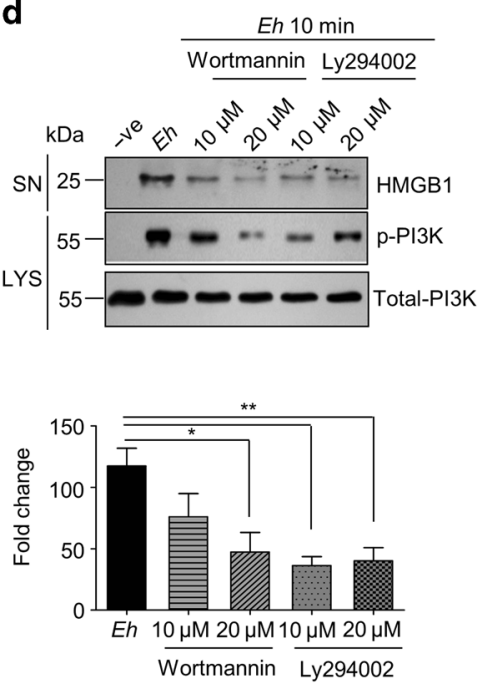

e
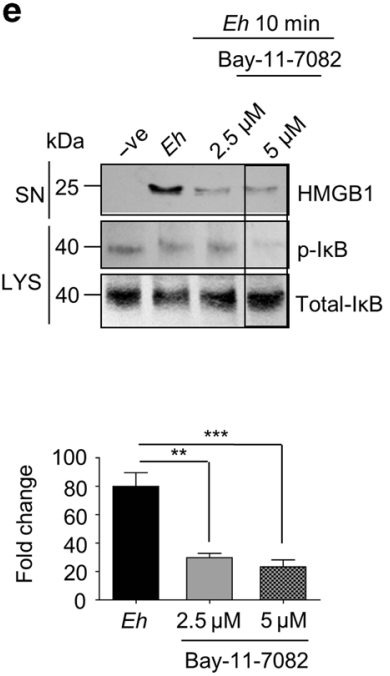

f
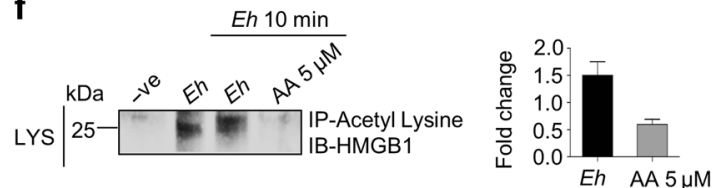

g

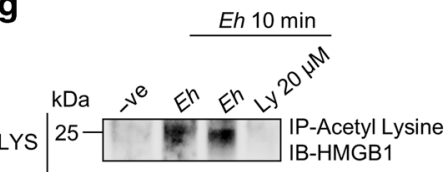

h

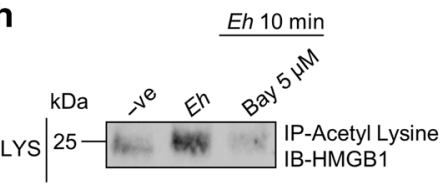

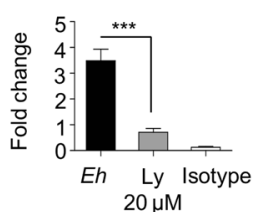

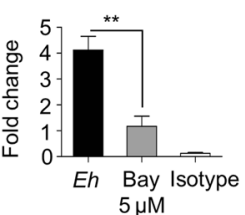

Fig. $5 \mathrm{PI} 3 \mathrm{~K}$ and NF-KB signaling pathways are required for the acetylation and secretion of HMGB1 in response to Eh. a THP-1 cells were incubated with $E$ for different times at 20:1 ratio and equal amounts of cell lysates (LYS) were loaded onto the SDS-PAGE gel to detect p-PI3K and p-ІкB levels. b Cytoplasmic extracts were prepared from Eh-stimulated and non-stimulated cells to detect levels of p-P65. c Eh were pretreated for $5 \mathrm{~min}$ with $55 \mathrm{mM}$ D-galactose before incubation with macrophages. THP-1 cells were stimulated with either galactose-treated Eh or Eh only for $10 \mathrm{~min}$ to assess p-PI3K and p-IKB levels. d THP-1 cells were pre-treated 30 min with Wortmannin $(10 \mu \mathrm{M}, 20 \mu \mathrm{M})$ and Ly294002 $(10 \mu \mathrm{M}, 20 \mu \mathrm{M})$ and stimulated with Eh for $10 \mathrm{~min}$. e THP-1 cells were pre-treated 30 min with Bay-11-7082 $(2.5 \mu \mathrm{M}, 5 \mu \mathrm{M})$ and stimulated with Eh for 10 min. Equal amounts of cell culture supernatant (SNs) were loaded onto SDS-PAGE to detect HMGB1. f-h THP-1 cells were pre-treated with AA $(5 \mu \mathrm{M})$, Ly294002 $(20 \mu \mathrm{M})$, or Bay-11-7082 $(5 \mu \mathrm{M})$ for 30 min and then stimulated with Eh for 10 min. Equal amounts of protein samples were prepared for the immunoprecipitation (IP) assay. Acetyl lysine antibody was used for immunoprecipitation and immunoblotted with anti-HMGB1 antibody. Normal rabbit IgG antibody was used as isotype control. Data are representative of three independent experiments. ${ }^{*} P<0.05,{ }^{* *} P<0.01$, and ${ }^{* *} P<0.001$. Bar represent mean $\pm \mathrm{SEM}$.

inflammasome and caspase-1 activation and independent of this well-characterized Eh-macrophage outside in signaling event. Most studies on Eh pathogenicity primarily focus on parasite rather than host factors such as ineffective or excessive innate immune responses. Inflammation is a two-edged sword: it can defend the host from invasive infections or it can trigger severe host tissue damage. Previous studies on the pathogenesis of amebic intestinal lesions in gerbils showed infiltration of inflammatory cells and destruction of the epithelium allowing Eh to invade. ${ }^{13}$ In this study, we showed that $E h$ induced rapid and early secretion of HMGB1 from macrophage and epithelial cells. Extracellular HMGB1 binds cell surface receptors in an autocrine and/or paracrine manner to elicit pro-inflammatory responses, ${ }^{30}$ and neutralization of HMGB1 significantly decreased proinflammatory cytokines/chemokines expression in vivo. These findings might explain the observed cellular infiltration and tissue destruction seen during acute intestinal amebiasis.

HMGB1 is also known as late mediator of endotoxin-induced lethality in mice. Murine models of endotoxemia or sepsis induced by cecal ligation puncture (CLP) showed first appearance of HMGB1 beginning at 6 to $8 \mathrm{~h}$ and highest detection at 16 to $32 \mathrm{~h}^{15,31}$ Mycobacterium tuberculosis infection in BMDM and RAW 264.7 cells induced HMGB1 secretion after $24 \mathrm{~h}$ of infection. ${ }^{32}$
Surprisingly, in contrast to these studies, we observed Eh-induced secretion of HMGB1 within $5 \mathrm{~min}$. Importantly, macrophage contact with live parasite via the Gal-lectin, but not secreted components or lysed $E h$, triggered the release of HMGB1. These results substantiates that adherence of live $E h$ to target cells is a critical component to evoke cellular responses ${ }^{14}$ and signaling events. Another important observation of our study was that purified native Gal-lectin alone stimulated modest HMGB1 secretion. We have previously shown that Gal-lectin activates NF-KB signaling pathway ${ }^{10,17}$ to stimulate TNF- $\alpha$ and IL12 production from macrophages. ${ }^{7,33}$ Therefore, Eh surface Gallectin or soluble Gal-lectin can act as a pathogen-associated molecular pattern (PAMP) to activate immune cells to generate potential immune responses. However, compared to the live Ehassociated Gal-lectin, soluble Gal-lectin was slow acting in naive macrophages. Active secretion of HMGB1 is mediated by acetylation of the NLS, which cause the protein to lose its interaction with the nucleus. ${ }^{18}$ This occurs when PAMPs and inflammatory mediators such as LPS and TNF-a activate immune cells. ${ }^{34-36}$ Post-translational alteration within NLS of intracellular proteins are critical to control the sub-cellular localization between the nucleus and the cytoplasm. ${ }^{37}$ HMGB1 have two NLS sites with the abundance of lysine residues that can easily undergo 
a

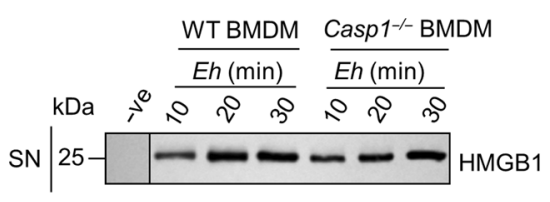

C

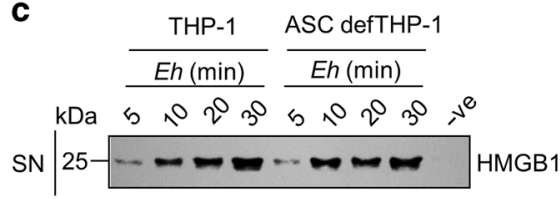

e

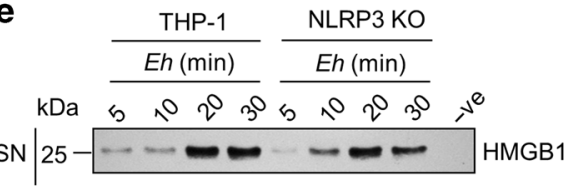

g

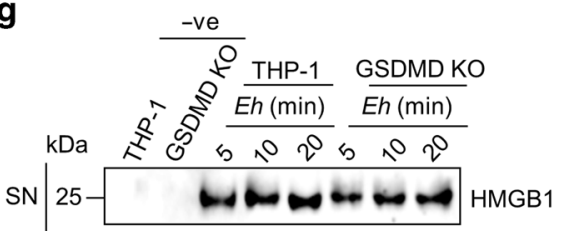

i

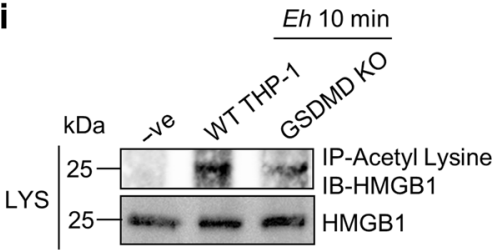

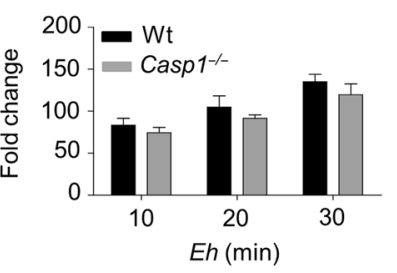
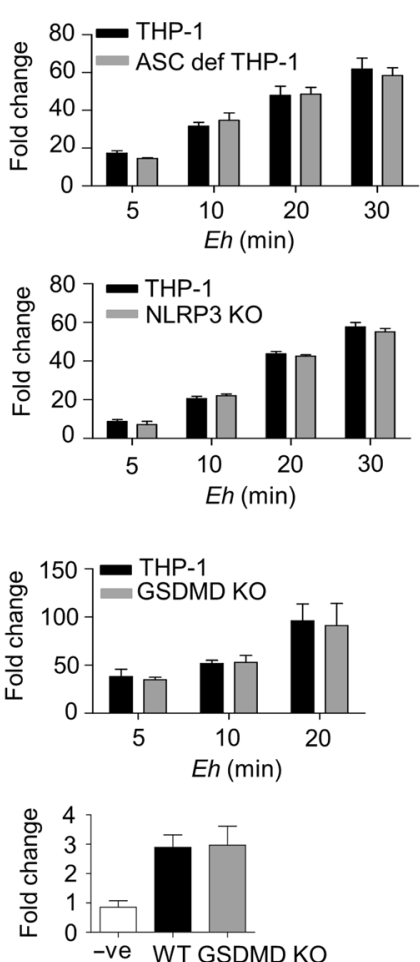

b

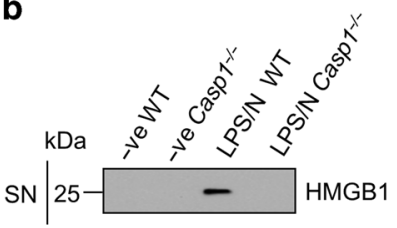

d

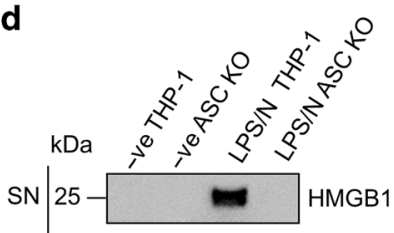

f

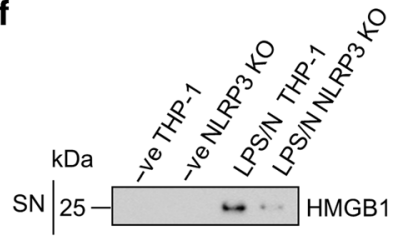

h

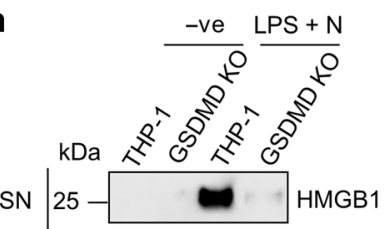

Fig. 6 Inflammasome and gasdermin D-independent release of HMGB1 in response to Eh. $\mathbf{a}, \mathbf{b}$ BMDMs from WT and Caspase ${ }^{-1-}$ mice were stimulated with Eh for different time points at 20:1 ratio and with LPS + nigericin (LPS $100 \mathrm{ng} / \mathrm{ml}$, nigericin $10 \mu \mathrm{M}$ ), an activator of NLRP3 inflammasome that leads to pyroptosis. c, d WT and ASC-deficient THP-1 macrophages were stimulated with Eh for different time points and with LPS + nigericin (LPS $100 \mathrm{ng} / \mathrm{ml}$, nigericin $10 \mu \mathrm{M}$ ). e, f WT and NLRP3 CRISPR/Cas9-KO THP-1 macrophages were stimulated with Eh for different time points and with LPS + nigericin (LPS $100 \mathrm{ng} / \mathrm{ml}$, nigericin $10 \mu \mathrm{M}$ ). Equal amounts of cell culture supernatants (SNs) were loaded onto the SDS-PAGE gel to detect HMGB1 level. $\mathbf{g}, \mathbf{h}$ WT and GSDMD CRISPR/Cas9-KO THP-1 macrophages were stimulated with Eh for different time points and with LPS + nigericin (LPS $100 \mathrm{ng} / \mathrm{ml}$, nigericin $10 \mu \mathrm{M})$. HMGB1 was detected in the supernatant by western blot and quantified by densitometric analysis. i WT and GSDMD CRISPR/Cas9-KO THP-1 macrophages were stimulated with Eh for 10 min and equal amounts of protein samples were prepared for immunoprecipitation assay. Acetyl lysine antibody was used for immunoprecipitation and immunoblotted with anti-HMGB1 antibody. Data are representative of three independent experiments. Bars represent mean \pm SEM.

acetylation during inflammation and cellular stress leading to nuclear-to-cytoplasmic translocation. ${ }^{18,38,39}$ In our study, Eh interaction with macrophages via the Gal-lectin-activated PI3K and NF-KB signaling pathways and triggered HAT activity to facilitate the acetylation of HMGB1 to translocate into the cytoplasm for subsequent release into the extracellular space.

Lethal endotoxemia or sepsis-induced extracellular HMGB1 release from activated immune cells was dependent on caspase- 1 and its upstream inflammasome activator components (ASC and NLRP3) and genetic deletion of inflammasome components reduce HMGB1 release. ${ }^{22,40}$ Interestingly, although Eh elicits NLRP3 inflammasome activation with the recruitment of active caspase- 1 by ASC component, ${ }^{14}$ Eh-induced HMGB1 release was independent of caspase-1, ASC, and NLRP3 inflammasome. Inflammasome-mediated HMGB1 release during endotoxemia was due to the pyroptotic cell death, ${ }^{21}$ which we also observed with LPS and nigericin stimulation. Nigericin along with LPS is a potent activator of NLRP3 inflammasome with caspase- 1 activation and subsequent induction of pyroptosis. ${ }^{41}$ The absence of inflammasome components completely reduced HMGB1 release with LPS and nigericin stimulation, whereas early secretion of Eh-induced HMGB1 occurred prior to caspase- 1 and inflammasome activation, and genetic deletion of inflammasome components had no effect on the HMGB1secretion. These results were supported by the LDH assays, which confirmed that HMGB1 secretion was independent of cell death. Other known signal peptide sequence-less protein is IL-1 $\beta$, and recently we have shown GSDMD pore-dependent extracellular transportation of IL-1 $\beta$ in the absence of pyroptosis. ${ }^{12,24}$ Based on these observations, we investigated the role of GSDMD pores in Eh induced extracellular release of HMGB1 and found GSDMD independent secretion of HMGB1. Interestingly, with LPS and nigericin stimulation, HMGB1 secretion was completely abolished in GSDMD CRISPR/Cas9-KO cells compared to the WT. Inflammasome activation with LPS and nigericin leads to rapid pore formation, destabilize osmotic balance, and results in cell lysis. Small proteins (such as IL-1 $\beta$ ) are released from cells before the cell rupture when pore formations are less/slower, leading to a stage called "macrophage hyperactivation." Dissimilarity between GSDMD-dependent IL-1 $\beta$ and HMGB1 secretion can be explained in two ways. First, IL-1 $1 \beta$ is expressed as a biologically inactive pro-form molecule, which is 

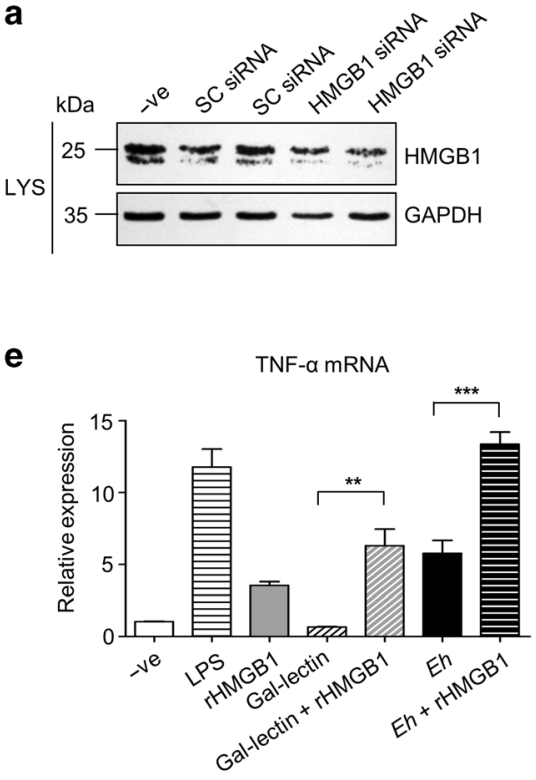

b

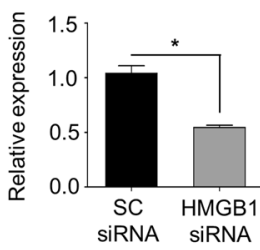

C

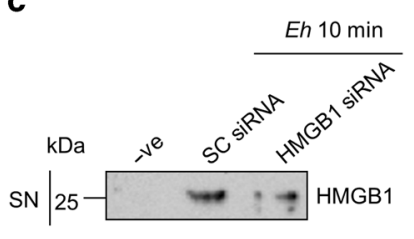

d TNF-a mRNA

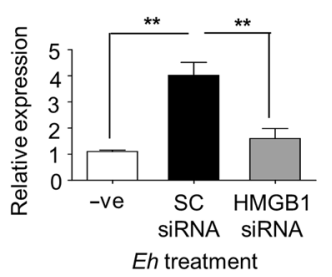

f
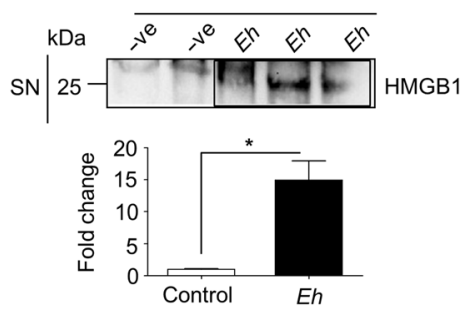

g

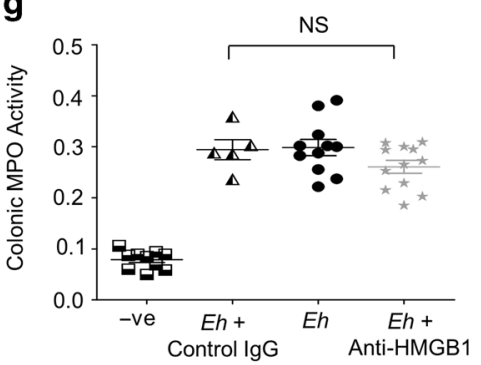

h

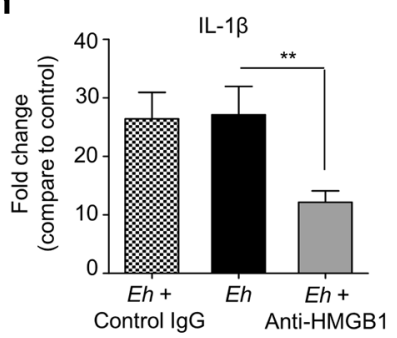

i

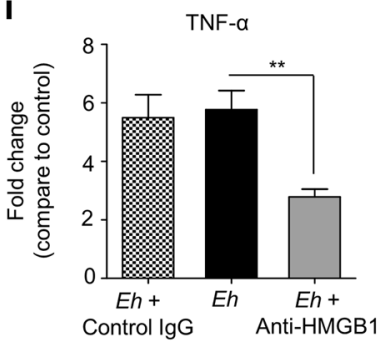

j

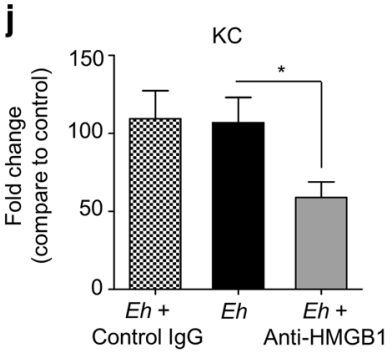

k

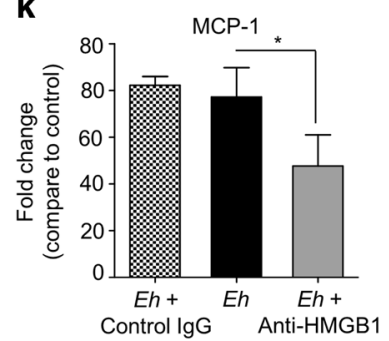

Fig. 7 In vitro silencing and in vivo neutralization of HMGB1 reduced pro-inflammatory cytokine expression in response to Eh. a, $\mathbf{b}$ THP-1 cells were transfected with $100 \mathrm{nM}$ of HMGB1 siRNA or scrambled siRNA. After $72 \mathrm{~h}$, transfected cells were stimulated with Eh. Silencing of HMGB1 was measured by western blot and mRNA expression. c Silencing of HMGB1 showed decreased secretion of HMGB1 in response to Eh as compared to the scrambled control. d Pro-inflammatory cytokine TNF- $\alpha$ mRNA expression was significantly decreased in HMGB1-silenced cells in response to Eh. e Recombinant HMGB1 (rHMGB1) alone or together with Eh or native Gal-lectin synergistically increased TNF- $\alpha$ mRNA expression as compared to only Eh or native Gal-lectin stimulation. LPS was used as a positive control. $\mathbf{f}$ In Eh-inoculated colonic loop study, the presence of HMGB1 in the luminal contents was quantified by western blot and densitometric analysis. g Myeloperoxidase (MPO) activity in colonic tissues of control (-ve) and inoculated with Eh that received lgG isotype control or anti-HMGB1-neutralizing antibody. h-k Proinflammatory cytokines and chemokines mRNA expression in colonic tissues inoculated with $E h$ from $\mathbf{g}$ above. $n=6-8 .{ }^{*} P<0.05,{ }^{* *} P<0.01$, and ${ }^{* * *} P<0.001$. Bar represent mean \pm SEM.

stored in the cytoplasm until the cell encounters stimulation for activation and secretion, ${ }^{25}$ whereas HMGB1 is a pre-formed nuclear protein, and upon activation it translocates from the nucleus to the cytoplasm and without further processing is released into the extracellular space. ${ }^{16}$ Thus, HMGB1 might be exported out of the cell before GSDMD pore formation (which also required processing). Second, the diameter of the GSDMD pores range from 10 to $20 \mathrm{~nm}$ in liposomes; thus, endogenously produced GSDMD pores might be smaller. ${ }^{42}$ The molecular diameter of IL-1 $\beta$ is $4 \mathrm{~nm}$ ( $17 \mathrm{kDa})$, which is sufficient to pass through GSDMD pores, whereas the diameter of $\mathrm{LDH}$, the cell death marker, is $8.7 \mathrm{~nm}(147 \mathrm{kDa})$ and too large to pass through the pores and thus released by cell lysis. ${ }^{25}$ In contrast to IL-1 $\beta$, although the molecular weight of HMGB1 is $25 \mathrm{kDa}$, it has a higher molecular diameter $(\sim 7.9 \mathrm{~nm})$ to fit through the pores. The larger diameter is due to self-dimers/tetramers formation by HMGB1 or due to association with nuclear proteins or components of the transcription factors. ${ }^{25}$ In our study, secreted HMGB1 appeared as a doublet of acetylated and non-acetylated forms and may explain GSDMD-independent secretion of HMGB1. The mechanisms of how HMGB1 is exported from the cytoplasm to the extracellular space in response to $E h$ remains unknown and we speculate that it might be via secretory vesicles or by microvesicles based on the distinct puncta nature of HMGB1.

In vivo study with HMGB1-knockout mouse could have provided direct association between HMGB1 and Eh pathogenesis. However this was not possible as global knockout of HMGB1 is lethal as the protein is critical for proper transcriptional control of different transcription factors. ${ }^{43}$ In endotoxemia, neutralizing antibodies against HMGB1 conferred protection against LPSinduced death of mice. ${ }^{15}$ Similarly, treatment with anti-HMGB1neutralizing antibodies rescued mice from lethal sepsis induced by CLP. ${ }^{31}$ Our results are in accordance with these observations, as mice injected with anti-HMGB1-neutralizing antibody before $E h$ inoculation in colonic loops induced less pro-inflammatory cytokine/chemokine expression as compared to non-injected mice. Similarly, siRNA silencing of HMGB1 showed significant reduction of TNF-a expression compared to control siRNA. It is well known that administration of HMGB1 itself increases production of pro-inflammatory cytokines. ${ }^{44}$ An interesting observation from our study was that rHMGB1 in the presence of $E h$ and/or Gal-lectin induced a synergistic increase in TNF-a mRNA expression. These findings substantiate that secreted HMGB1 in response to Eh can act synergistically to augment pro-inflammatory responses in vivo. Consistent with these findings, neutralizing HMGB1 antibody 
a

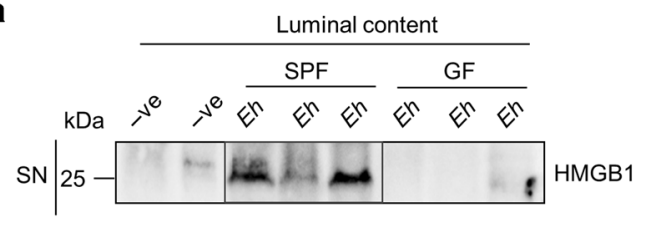

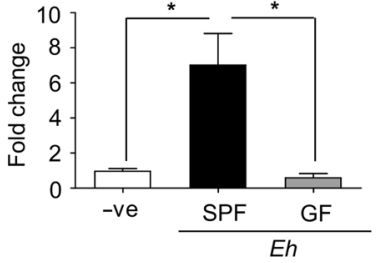

b

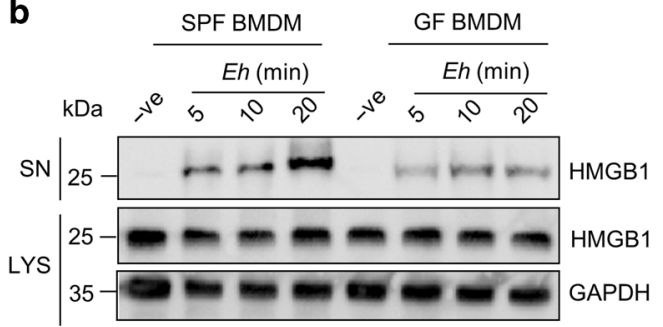

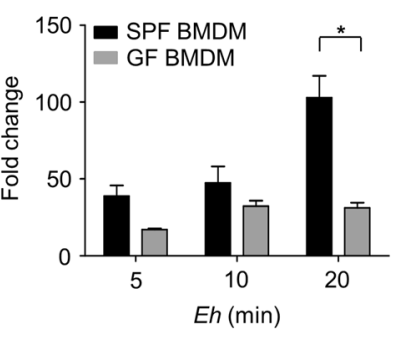

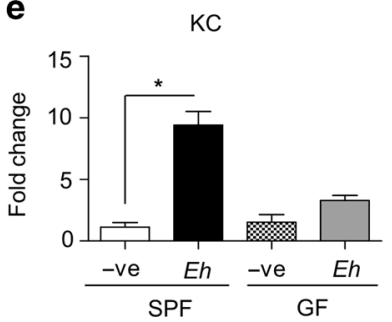

Fig. 8 Eh-induced HMGB1 release is dependent on microbiota. a Eh-inoculated colonic loops of SPF and GF mice were quantified for the presence of HMGB1 in luminal contents by western blot and densitometric analysis. b BMDM cells from SPF and GF mice were stimulated with Eh for different time points and equal amounts of cell culture supernatant (SNs) and cell lysates (LYS) were loaded onto SDS-PAGE to detect HMGB1. c-e Pro-inflammatory cytokine mRNA expression for IL-1 $\beta$, TNF- $\alpha$ and KC were determined from SPF and GF mice colonic loop tissues. Data are representative of three independent experiments. Bar represent mean \pm SEM. ${ }^{*} P<0.05$.

during Eh-induced inflammation in colonic loops significantly inhibited pro-inflammatory cytokine and chemokine expression, but was not sufficient to reduce MPO activity during the $3 \mathrm{~h}$ infection.

Because of the wide variation in the presentation of Eh pathogenicity, an emerging body of work proposed a role for host intestinal microbiota in the development and outcome of infection. Recent studies suggest that microbiota shapes the immune system and modulates bone marrow processes that give rise to immune effector cells required for pathogen clearance. ${ }^{45}$ As a result, GF mice are impaired in boosting immune responses as well as failed to clear infection. ${ }^{29,45}$ Recent studies have shown that colonization of mice with Clostidia, segmented filamentous bacteria, alters bone marrow cells and increases host damageassociated molecular pattern molecule serum amyloid $A$ into the serum compared to non-colonized mice and provides protection against $E$ infection. ${ }^{28,46}$ Our results are in accordance with these observations, as significant levels of HMGB1 was detected in the luminal content of SPF mice inoculated with Eh but not in GF mice. Thus, secretion of host HMGB1, which is essential for augmented inflammatory responses, are microbiota dependent. These results highlight the importance of microbiota in immune system development as well as in endogenous alarmin molecules secretion.

In summary, we have shown that Eh Gal-lectin-mediated interaction with macrophages is the key event that activates $\mathrm{PI} 3 \mathrm{~K} / \mathrm{NF}-\mathrm{KB}$ signaling pathways and induced HAT activity to promote acetylation and translocation of nuclear HMGB1 to the cytoplasm. In the absence of pyroptosis, cytoplasmic HMGB1 export to the extracellular space was independent of NLRP3 inflammasome activation and GSDMD pores. In Eh infection, HMGB1 played a critical role in augmenting pro-inflammatory responses that was developmentally regulated by intestinal microbiota. As the current knowledge of extracellular HMGB1 secretion is limited on endotoxemia-mediated inflammasome activation and pyroptotic cell lysis, our study highlights the existence of different mechanisms that regulate HMGB1 secretion in response to a parasite rather than cell lysis.

\section{METHODS}

Entamoeba histolytica culture

Virulent strains of Eh HM-1:IMSS were grown axenically in TYI-S-33 medium along with $100 \mathrm{U} / \mathrm{ml}$ penicillin and $100 \mu \mathrm{g} / \mathrm{ml}$ streptomycin sulfate at $37^{\circ} \mathrm{C}$ in sealed borosilicate glass tubes as described previously. ${ }^{47}$ Eh were harvested after $72 \mathrm{~h}$ of growth (log phase) by keeping on ice for 5 min before centrifugation at $200 \times g$ for $5 \mathrm{~min}$ at $4{ }^{\circ} \mathrm{C}$. After centrifugation, Eh were resuspended in serumfree RPMI to $1 \times 10^{6} \mathrm{Eh} / \mathrm{ml}$.

Cell preparation and stimulation

THP-1 human monocytic cell line (ATCC) were cultured in RPMI supplemented with $10 \%$ fetal bovine serum (FBS), $10 \mathrm{mM}$ HEPES, $50 \mu \mathrm{M}$ 2-mercaptoethanol, $100 \mathrm{U} / \mathrm{ml}$ penicillin, and $100 \mu \mathrm{g} / \mathrm{ml}$ streptomycin sulfate in a cell culture incubator with $5 \% \mathrm{CO}_{2}$. To differentiate THP- 1 cells to macrophages, $8 \times 10^{5}$ cells per well were plated in 12-well plate in complete medium supplemented with $50 \mathrm{ng} / \mathrm{ml}$ phorbol-12-myristate-13-acetate (PMA) for overnight. BMDMs were cultured from the bone marrow of C57BL/6 mice or indicated knockout mice and grown for 6 days in complete medium supplemented with $30 \%$ L-929 SNs. Then, $8 \times$ $10^{5}$ cells/well were plated in 12-well plates with complete RPMI medium. Human T84 epithelial cell lines (ATCC) were cultured in Dulbecco's modified Eagle's medium F12 (1:1) supplemented with 


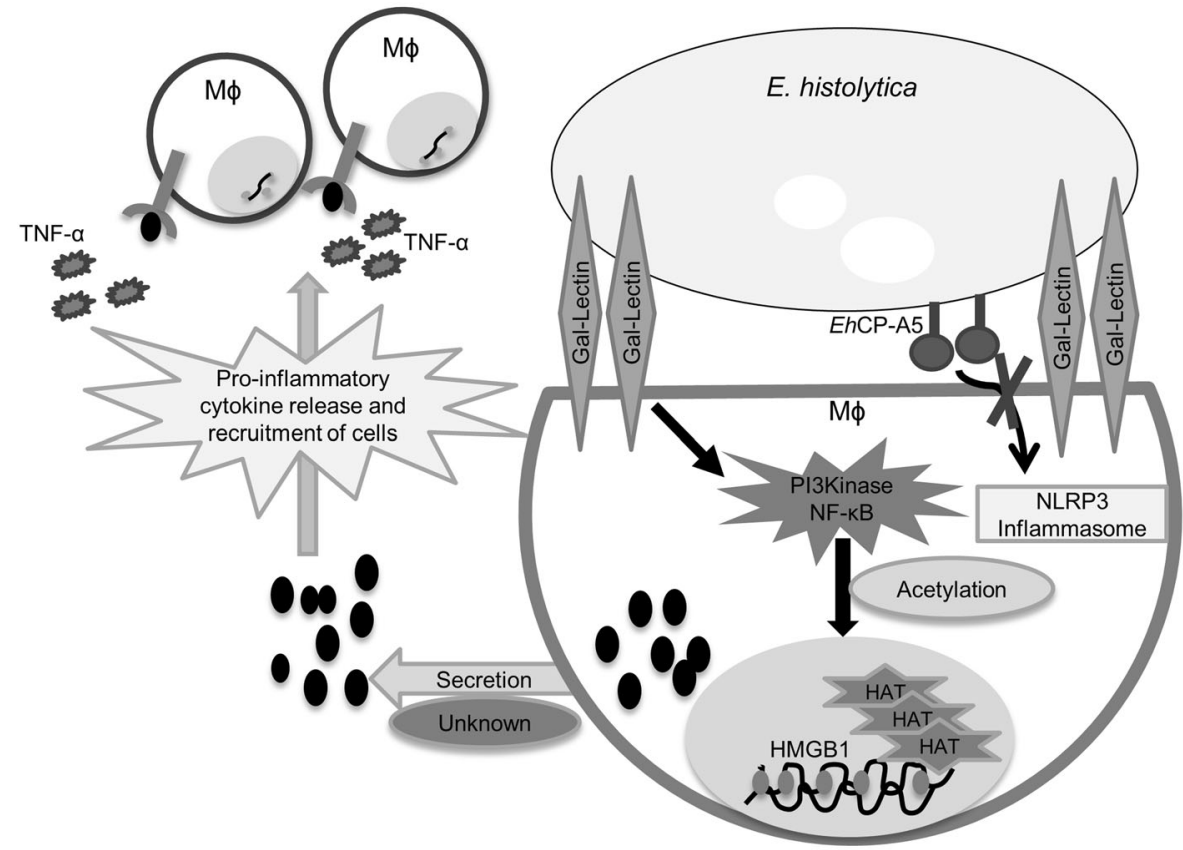

Fig. 9 Schematic representation of Eh Gal-lectin-mediated interaction with macrophages and regulated secretion of HMGB1. During Eh infection, contact with host cell is mediated by Eh surface Gal-lectin. Gal-lectin interaction leads to the activation of PI3K and NF- $\mathrm{KB}$ signaling pathways along with the activation of histone acetyltransferase (HAT) enzyme activity. HAT initiates the acetylation process within nuclear HMGB1 protein that loses its interaction with the nucleus. Acetylated HMGB1 translocate into the cytoplasm and subsequently released into the extracellular space to activate pro-inflammatory responses and recruit inflammatory cells to the site of infection. Interestingly, unlike endotoxemia, Eh surface cysteine protease EhCP-A5 mediated NLRP3 inflammasome activation is not required for active HMGB1 secretion.

$10 \% \mathrm{FBS}, 100 \mathrm{U} / \mathrm{ml}$ penicillin, and $100 \mu \mathrm{g} / \mathrm{ml}$ streptomycin sulfate in a cell culture incubator with $5 \% \mathrm{CO}_{2}$. For experiments, $3 \times 10^{5}$ cells were plated in 12-well plates and grown to $80 \%$ cell confluence.

For Eh stimulation, cells were treated at a 20:1 ratio of macrophage to $E h$ at $37^{\circ} \mathrm{C}$ in $400 \mu$ l serum-free RPMI/well for the indicated times. Cell culture SNs from three wells were pooled together for trichloroacetic acid (TCA) precipitation and for cell lysate, washed with cold PBS on ice, and lysed with lysis buffer ( $1 \%$ Triton X-100, $20 \mathrm{mM}$ Tris, $100 \mathrm{mM} \mathrm{NaCl}, 1 \mathrm{mM}$ EDTA, $200 \mathrm{mM}$ orthovanadate, sodium fluoride, $0.1 \%$ sodium dodecyl sulfate (SDS), phenylmethanesulfonyl fluoride, leupeptin, aprotinin, and protease inhibitor cocktail). For the Gal-lectin adhesion inhibition assay, $55 \mathrm{mM}$ exogenous D-galactose was incubated with Eh for $5 \mathrm{~min}$ at $37^{\circ} \mathrm{C}$ before stimulating THP-1 macrophages. For the inhibitor experiments, PMA-differentiated THP-1 macrophages were pre-treated with indicated inhibitors for $30 \mathrm{~min}$ at $37^{\circ} \mathrm{C}$ in serum-free RPMI prior to Eh stimulation. LPS from E. coli 0111:B4, PMA, and nigericin (N7143) were obtained from Sigma. Wortmannin, Ly294002, and AA were from Cayman Chemical. Bay-11-7082 was from Invivogen.

ASCdef THP-1, NLRP3, and GSDMD CRISPR/Cas9 THP-1-KO cells ASCdef THP-1 cells were purchased from Invivogen (thp-dasc). These cells were originally obtained from THP-human monocytic cells with no expression of ASC, but express basal levels of other inflammasome components. NLRP3 CRISPR/Cas9-KO THP-1 cells were a generous gift from Dr. Muruve (Department of Immunology, University of Calgary, Canada). GSDMD CRISPR/Cas9-KO THP1 cells were a generous gift from Dr. Bachovchin (Memorial Sloan Kettering Cancer Center, New York, USA).

Preparation of amebic secretory proteins, whole lysate, cytosolic, and membrane components

SPs of Eh were collected from $2 \times 10^{7}$ cells $/ \mathrm{ml}$ and then incubated with Hank's balanced salt solution (HBSS) for $2 \mathrm{~h}$ at $37^{\circ} \mathrm{C}$. Whole amebic lysates were prepared by freeze-thaw lysis of Eh in HBSS. Cytosolic and membrane components were prepared by centrifugation of freeze-thawed $E h$ lysates at $4{ }^{\circ} \mathrm{C}$ for 15 min at $14,000 \times$ g. The SN was designated as the cytosolic fraction and the resuspended pellet was the membrane fraction. Purified native Gal-lectin was a gift from Dr. David Lyerly (Techlab, Blacksburg, Virginia).

\section{Western blots}

THP-1 or BMDM SNs from three wells were pooled and concentrated by TCA precipitation. Precipitated SNs were suspended in $25 \mathrm{mM}$ Tris plus $1 \mathrm{~N} \mathrm{NaOH}$ and equal amounts of TCA-precipitated SNs and cell lysates were loaded on SDSpolyacrylamide gel electrophoresis and transferred onto nitrocellulose membrane, followed by blocking with $5 \%$ skim milk or $3 \%$ bovine serum albumin for phospho proteins. The blot was incubated overnight at $4{ }^{\circ} \mathrm{C}$ with specific primary antibodies and the next day appropriate secondary horse radish peroxidaseconjugated antibody was added. Protein bands were visualized with either SuperSignal Chemiluminescence Reagents (Pierce Biotechnology) or ChemiLucent ECL detection (EMD Millipore). Primary antibodies were: human anti-HMGB1 (R \&D systems), mouse anti-HMGB1 (ab18256), anti-phospho-PI3K (Cell Signaling, 4228), total anti-PI3K p55 (Cell Signaling, 11889), anti-phospho-IKB (Cell Signaling, 9246), total anti-IKB (Cell Signaling, 4812), and antiGAPDH (Millipore, Sigma).

Immunofluorescence

BMDM cells were harvested on coverslips for 6 days and stimulated with Eh for $10 \mathrm{~min}$. After stimulation, cells were washed gently with PBS and fixed with $3 \%$ paraformaldehyde for $10 \mathrm{~min}$. Cells were permeabilized with $0.01 \%$ Triton in PBS for $5 \mathrm{~min}$, followed by blocking with $2 \%$ donkey serum for $1 \mathrm{~h}$. Cells were stained with mouse anti-HMGB1 antibody overnight at $4{ }^{\circ} \mathrm{C}$. The next day, cells were washed gently with PBS-Tween $(0.1 \%)$ and incubated with specific secondary antibody and DAPI (4',6- 
Table 1. Primer sequences used for quantitative real-time PCR.

\begin{tabular}{|c|c|c|}
\hline Gene name & Sequences & Annealing $\left({ }^{\circ} \mathrm{C}\right)$ \\
\hline \multirow[t]{2}{*}{ Human HMGB1 } & F: GCAAGCGAACAGCAGGGTTA & 63 \\
\hline & R: TCAGATTGAGTCATTTGCTCCTCTT & \\
\hline \multirow[t]{2}{*}{ Human TNF-a } & F: AAGCCTGTAGCCCATGTTGT & 65 \\
\hline & R: GAGGTACAGGCCCTCTGATG & \\
\hline \multirow[t]{2}{*}{ Human GAPDH } & F: GGATTTGGTCGTATTGGG & 63 \\
\hline & R: GGAAGATGGTGATGGGATT & \\
\hline \multirow[t]{2}{*}{ Murine TNF-a } & F: ATGAGCACAGAAAGCATGATC & 56 \\
\hline & R: TACAGGCTTGTCACTCGAATT & \\
\hline \multirow[t]{2}{*}{ Murine IL-1 $\beta$} & F: GCCTCGTGCTGTCGGACCCA & 58 \\
\hline & R: CTGCAGGGTGGGTGTGCCGT & \\
\hline \multirow[t]{2}{*}{ Murine KC } & F: ACCCAAACCGAAGTCATAGC & 60 \\
\hline & R: TCTCCGTTACTTGGGGACAC & \\
\hline \multirow[t]{2}{*}{ Murine $M C P-1$} & F: AGCCAACTCTCACTGAAG & 58 \\
\hline & R: TCTCCAGCCTACTCATTG & \\
\hline \multirow[t]{2}{*}{ Murine Actin } & F: CTACAATGAGCTGCGTGTG & 54 \\
\hline & R: TGGGGTGTTGAAGGTCTC & \\
\hline
\end{tabular}

diamidino-2-phenylindole) to stain nuclei at room temperature for $1 \mathrm{~h}$. The coverslips were washed and mounted onto microscope slides with FluoroSave ${ }^{\mathrm{TM}}$ reagent (Calbiochem) and imaged using Olympus IX81 FV1000 Fluoview Laser Scanning Confocal microscope with a $\times 60$ objective. Image preparation and quantification of the cytoplasmic HMGB1 intensity was performed using the ImageJ software.

\section{Immunoprecipitation}

Anti-acetyl lysine antibody was used to pull down proteins from cell lysate in the presence of $10 \%$ A/G beads (Santa Cruz) at $4{ }^{\circ} \mathrm{C}$ overnight. Protein-bead complexes were washed 5-6 times with lysis buffer and samples were boiled at $95^{\circ} \mathrm{C}$ for $5 \mathrm{~min}$. Immunoblots were done using anti-HMGB1 antibody.

HMGB1 siRNA

PMA-differentiated THP-1 cells were transfected with HMGB1 siRNA $(100 \mathrm{nM})$ or scramble siRNA as a control (Dharmacon, On target plus SMART pool siRNA), with the INTERFERin reagent (409-10, Polyplus) according to the manufacturer's protocol. For each transfection, either with HMGB1 siRNA or scramble siRNA, a total of $7 \mu \mathrm{l}$ INTERFERin reagent was used. Every $24 \mathrm{~h}$ media were replaced with new complete RPMI. After $72 \mathrm{~h}$ following siRNA transfection, cells were stimulated with Eh.

LDH assay and HAT activity assay

The release of $\mathrm{LDH}$ from macrophages was measured by using Promega $^{\mathrm{TM}}$ Cytox-ONE homogeneous membrane integrity assay (G7890, Fisher Scientific) according to the manufacturer's instruction. HAT activity was measured by using HAT Activity Colorimetric Assay Kit (K332, Biovision) according to the manufacturer's instruction.

Gene expression analysis by real-time qPCR

Total RNA was extracted by using E.Z.N.A. ${ }^{\circledR}$ Total RNA Kit (Omega BioTEK) from cells and from snap-frozen tissue using Trizol reagent protocol (Invitrogen; Life Technologies, Burlington,ON) as per the manufacturer's instructions. The purity and yield of the extracted RNA was measured by the ratio of absorbance at $260 / 280 \mathrm{~nm}$ (NanoDrop, Thermo Scientific). Complementary DNA (cDNA) preparation done by using qScript cDNA Synthesis Kit. Real-time quantitative PCR was performed using a Rotor Gene 3000 Realtime PCR System (Corbett Research). Each reaction mixture contained 1:10 dilution of CDNA, SYBR Green PCR Master Mix (Qiagen) and $1 \mu \mathrm{M}$ of primers $(F+R)$. The results were analyzed using the $2^{-\triangle \Delta C T}$ methods and expressed as fold changes. A complete list of the primer sequences and conditions used are listed in Table 1.

Animals

C57BL/6 mice were obtained from Charles River. Caspase $1^{-/-}$mice on a C57BL/ 6 background were obtained from Dr. Yan Shi (University of Calgary) and N/rp3 $3^{-/-}$on a C57BL/6 background were obtained from Dr. Dan Muruve (University of Calgary). GF mice on a C57BL/6 background were purchased from the International Microbiome Center at the University of Calgary.

In vivo colonic loop with $E$ infection

Colonic loops were used as a short-term infection model of Eh as previously described. ${ }^{27}$ Briefly, mice were anesthetized, and following laparotomy, the colon was exteriorized and a ligation with 3-0 black silk sutures (Ethicon, Somerville, NJ; Peterborough, ON, Canada) was done at the proximal (distal to the cecum) and distal (proximal to the rectum) ends with care to keep the mesenteries, blood vessels, and nerves intact. Virulent log-phase WT trophozoites $\left(1 \times 10^{6}\right)$ in $100 \mu$ PBS were inoculated in the colonic loop. The control group was inoculated with $100 \mu \mathrm{l}$ PBS. After $3 \mathrm{~h}$, luminal contents were collected and tissues were excised and fixed in Carnoy's, embedded in paraffin and $5 \mu \mathrm{m}$ sections stained with hematoxylin and eosin for inflammatory cellular infiltrates. Other tissues were processed for RNA and MPO activity. Equal volume of luminal content protein was used for TCA precipitation and for western blotting. To neutralize HMGB1, animals were injected i.p. with $75 \mu \mathrm{g}$ per mouse-neutralizing antibodies (mouse monoclonal 3E8, BioLegend) or control IgG $12 \mathrm{~h}$ before $E h$ inoculation in colonic loop. For intestinal permeability assay, $3 \mathrm{~h}$ before the colonic loop, mice were gavaged with $15 \mathrm{mg}$ of fluorescein isothiocyanate-dextran (3-5 $\mathrm{kDa}$, Sigma-Aldrich) dissolved in $100 \mu \mathrm{l}$ of water. Following $3 \mathrm{~h}$ colonic loop study with Eh inoculation, blood was collected by cardiac puncture and was allowed to clot in the dark for $3 \mathrm{~h}$ at room temperature. After centrifugation at $10,000 \times g$ for $10 \mathrm{~min}$, serum was transferred to a clean Eppendorf tube, and diluted with PBS. One hundred microliters of diluted samples were loaded onto a black bottom 96-well plates and fluorescence was determined (absorption $485 \mathrm{~nm}$, emission $535 \mathrm{~nm}$ ).

\section{Statistics}

All experiments are representative of at least three independent experiments. Densitometry analysis was performed by the Image Lab software. Statistical significance between two groups was done by Student's $t$ test and comparison between two or more groups were done by one-way analysis of variance (ANOVA), followed by post hoc Bonferroni test. GrapPad Prism5 was used for these statistical analysis and $P<0.05$ was considered as significant. For the confocal image quantification, a minimum of 7 images was used for each condition and with same brightness adjustment. Results were shown as the mean \pm SEM.

\section{ACKNOWLEDGEMENTS}

We thank Dr. Y. Shi for the Caspase $1^{-/-}$mice, Dr. D. Muruve for the NLRP3 CRISPR-KO cells and NIrp3 ${ }^{-/-}$mice, and Dr. D. Bachovchin for the GSDMD CRISPR-KO cells. This work was funded by a Discovery Grant (RGPIN-2014-04023) from the Natural Sciences and Engineering Research Council of Canada awarded to K.C. The funders had no role in study design, data collection and analysis, decision to publish, or preparation of the manuscript. 


\section{AUTHOR CONTRIBUTIONS}

S.B. and K.C. constructed the experimental design. K.C. and F.M. contributed reagents and methodical support. S.B. performed the majority of experiments and analysis of data. F.M. performed the parasite cultivation and F.M and A.L.C contributed with in vivo mouse colonic loop experiments. S.B. and K.C. wrote the manuscript.

\section{ADDITIONAL INFORMATION}

The online version of this article (https://doi.org/10.1038/s41385-019-0233-6) contains supplementary material, which is available to authorized users.

Competing interests: The authors declare no competing interests.

Ethics approval: The Health Sciences Animal Care Committee from the University of Calgary have examined the animal care and treatment protocol (AC18-0218) and approved the experimental procedures proposed and verifies with the applicant that the care and treatment of animals used was in agreement with the principles outlined in the most recent policies on the "Guide to the Care and Use of Experimental Animals" by The Canadian Council on Animal Care.

Publisher's note Springer Nature remains neutral with regard to jurisdictional claims in published maps and institutional affiliations.

\section{REFERENCES}

1. Stanley, S. L. Jr Amoebiasis. Lancet 361, 1025-1034 (2003).

2. Mortimer, L. \& Chadee, K. The immunopathogenesis of Entamoeba histolytica. Exp. Parasitol. 126, 366-380 (2010)

3. Haque, R., Huston, C. D., Hughes, M., Houpt, E. \& Petri, W. A. Jr Amebiasis. N. Engl. J. Med. 348, 1565-1573 (2003).

4. St-Pierre, J. et al. The macrophage cytoskeleton acts as a contact sensor upon interaction with Entamoeba histolytica to trigger IL-1 $\beta$ secretion. PLoS Pathog. 13, e1006592 (2017).

5. Mortimer, L., Moreau, F., Cornick, S. \& Chadee, K. The NLRP3 inflammasome is a pathogen sensor for invasive Entamoeba histolytica via activation of a5 $\beta 1$ integrin at the macrophage-amebae intercellular junction. PLoS Pathog. 11, e1004887 (2015).

6. Lin, J. \& Chadee, K. Macrophage cytotoxicity against Entamoeba histolytica trophozoites is mediated by nitric oxide from L-arginine. J. Immunol. 148, 3999-4005 (1992).

7. Seguin, R., Mann, B. J., Keller, K. \& Chadee, K. Identification of the galactoseadherence lectin epitopes of Entamoeba histolytica that stimulate tumor necrosis factor-alpha production by macrophages. Proc. Natl Acad. Sci. USA 92, 12175-12179 (1995).

8. Lin, J.-Y., Seguin, R., Keller, K. \& Chadee, K. Tumor necrosis factor alpha augments nitric oxide-dependent macrophage cytotoxicity against Entamoeba histolytica by enhanced expression of the nitric oxide synthase gene. Infect. Immun. 62, 1534-1541 (1994)

9. Haque, R. et al. Correlation of interferon- $\gamma$ production by peripheral blood mononuclear cells with childhood malnutrition and susceptibility to amebiasis. Am. J. Trop. Med. Hyg. 76, 340-344 (2007).

10. Kammanadiminti, S. J., Mann, B. J., Dutil, L. \& Chadee, K. Regulation of Toll-like receptor-2 expression by the Gal-lectin of Entamoeba histolytica. FASEB J. 18, 155-157 (2004).

11. Moonah, S. N., Jiang, N. M. \& Petri, W. A. Jr Host immune response to intestinal amebiasis. PLoS Pathog. 9, e1003489 (2013).

12. Quach, J., Moreau, F., Sandall, C. \& Chadee, K. Entamoeba histolytica-induced IL-1 $\beta$ secretion is dependent on caspase-4 and gasdermin D. Mucosal Immunol. 12, 323-339 (2018)

13. Chadee, K. \& Meerovitch, E. Entamoeba histolytica: early progressive pathology in the cecum of the gerbil (Meriones unguiculatus). Am. J. Trop. Med. Hyg. 34, 283-291 (1985).

14. Mortimer, L., Moreau, F., Cornick, S. \& Chadee, K. Gal-lectin-dependent contact activates the inflammasome by invasive Entamoeba histolytica Mucosal Immunol. 7, 829-841 (2014).

15. Wang, H. et al. HMG-1 as a late mediator of endotoxin lethality in mice. Science 285, 248-251 (1999).

16. Ulloa, L. \& Messmer, D. High-mobility group box 1 (HMGB1) protein: friend and foe. Cytokine Growth Factor Rev. 17, 189-201 (2006).

17. Ivory, C. P. \& Chadee, K. Activation of dendritic cells by the Gal-lectin of Entamoeba histolytica drives Th1 responses in vitro and in vivo. Eur. J. Immunol. 37, 385-394 (2007).
18. Bonaldi, T. et al. Monocytic cells hyperacetylate chromatin protein HMGB1 to redirect it towards secretion. EMBO J. 22, 5551-5560 (2003).

19. Polevoda, B. \& Sherman, F. The diversity of acetylated proteins. Genome Biol. 3, reviews0006.0001 (2002)

20. Cornick, S., Moreau, F. \& Chadee, K. Entamoeba histolytica cysteine proteinase 5 evokes mucin exocytosis from colonic goblet cells via av $\beta 3$ integrin. PLoS Pathog. 12, e1005579 (2016)

21. Lu, B., Wang, H., Andersson, U. \& Tracey, K. J. Regulation of HMGB1 release by inflammasomes. Protein Cell. 4, 163-167 (2013).

22. Lamkanfi, M. et al. Inflammasome-dependent release of the alarmin HMGB1 in endotoxemia. J. Immunol. 185, 4385-4392 (2010).

23. Lopez-Castejon, G. \& Brough, D. Understanding the mechanism of IL-1beta secretion. Cytokine Growth Factor Rev. 22, 189-195 (2011).

24. Evavold, C. L. et al. The pore-forming protein gasdermin D regulates interleukin-1 secretion from living macrophages. Immunity 48, 35-44.e36 (2018).

25. Heilig, R. et al. The gasdermin-D pore acts as a conduit for IL-1beta secretion in mice. Eur. J. Immunol. 48, 584-592 (2018).

26. van Zoelen, M. A. et al. Role of toll-like receptors 2 and 4, and the receptor for advanced glycation end products in high-mobility group box 1-induced inflammation in vivo. Shock 31, 280-284 (2009).

27. Kissoon-Singh, V., Moreau, F., Trusevych, E. \& Chadee, K. Entamoeba histolytica exacerbates epithelial tight junction permeability and proinflammatory responses in Muc2(-/-) mice. Am. J. Pathol. 182, 852-865 (2013).

28. Burgess, S. L. \& Petri, W. A. Jr The intestinal bacterial microbiome and E. histolytica infection. Curr. Trop. Med. Rep. 3, 71-74 (2016).

29. Leon-Coria, A., Kumar, M., Moreau, F. \& Chadee, K. Defining cooperative roles for colonic microbiota and Muc2 mucin in mediating innate host defense against Entamoeba histolytica. PLoS Pathog. 14, e1007466 (2018).

30. Erlandsson Harris, H. \& Andersson, U. Mini-review: the nuclear protein HMGB1 as a proinflammatory mediator. Eur. J. Immunol. 34, 1503-1512 (2004).

31. Yang, H. et al. Reversing established sepsis with antagonists of endogenous highmobility group box 1. Proc. Natl. Acad. Sci. USA 101, 296-301 (2004).

32. Grover, A. et al. Mycobacterial infection induces the secretion of high-mobility group box 1 protein. Cell Microbiol. 10, 1390-1404 (2008).

33. Campbell, D., Mann, B. J. \& Chadee, K. A subunit vaccine candidate region of the Entamoeba histolytica galactose-adherence lectin promotes interleukin-12 gene transcription and protein production in human macrophages. Eur. J. Immunol. 30, 423-430 (2000).

34. Yanai, H. et al. HMGB proteins function as universal sentinels for nucleic-acidmediated innate immune responses. Nature 462, 99 (2009).

35. Lu, B. et al. JAK/STAT1 signaling promotes HMGB1 hyperacetylation and nuclear translocation. Proc. Natl. Acad. Sci. USA 111, 3068-3073 (2014).

36. Gardella, S. et al. The nuclear protein HMGB1 is secreted by monocytes via a nonclassical, vesicle-mediated secretory pathway. EMBO Rep. 3, 995-1001 (2002).

37. Yang, H., Antoine, D. J., Andersson, U. \& Tracey, K. J. The many faces of HMGB1: molecular structure-functional activity in inflammation, apoptosis, and chemotaxis. J. Leukoc. Biol. 93, 865-873 (2013).

38. Zhang, X. et al. Calcium/calmodulin-dependent protein kinase (CaMK) IV mediates nucleocytoplasmic shuttling and release of HMGB1 during lipopolysaccharide stimulation of macrophages. J. Immunol. 181, 5015-5023 (2008).

39. Evankovich, J. et al. High mobility group box 1 release from hepatocytes during ischemia and reperfusion injury is mediated by decreased histone deacetylase activity. J. Biol. Chem. 285, 39888-39897 (2010).

40. Lu, B. et al. Novel role of PKR in inflammasome activation and HMGB1 release. Nature 488, 670-674 (2012)

41. Mariathasan, S. et al. Cryopyrin activates the inflammasome in response to toxins and ATP. Nature 440, 228-232 (2006).

42. Fink, S. L. \& Cookson, B. T. Caspase-1-dependent pore formation during pyroptosis leads to osmotic lysis of infected host macrophages. Cell Microbiol. 8, 1812-1825 (2006).

43. Calogero, S. et al. The lack of chromosomal protein $\mathrm{Hmg} 1$ does not disrupt cell growth but causes lethal hypoglycaemia in newborn mice. Nat. Genet. 22, 276 (1999).

44. Abraham, E., Arcaroli, J., Carmody, A., Wang, H. \& Tracey, K. J. HMG-1 as a mediator of acute lung inflammation. J. Immunol. 165, 2950-2954 (2000).

45. Khosravi, A. et al. Gut microbiota promote hematopoiesis to control bacterial infection. Cell Host Microbe 15, 374-381 (2014).

46. Burgess, S. L. et al. Bone marrow dendritic cells from mice with an altered microbiota provide interleukin 17A-dependent protection against Entamoeba histolytica colitis. MBio 5, e01817 (2014).

47. Diamond, L. S., Harlow, D. R. \& Cunnick, C. C. A new medium for the axenic cultivation of Entamoeba histolytica and other Entamoeba. Trans. R. Soc. Trop. Med. Hyg. 72, 431-432 (1978). 